OPEN ACCESS

Edited by:

Denis Lacroix

UMR8608 Institut de Physique Nucléaire d'Orsay (IPNO), France

Reviewed by:

Marcella Grasso,

UMR8608 Institut de Physique

Nucléaire d'Orsay (IPNO), France Gianluca Colò,

University of Milan, Italy

*Correspondence:

Mitsuru Tohyama

tohyama@ks.kyorin-u.ac.jp

Specialty section: This article was submitted to Nuclear Physics,

a section of the journal

Frontiers in Physics

Received: 22 November 2019 Accepted: 28 February 2020

Published: 25 March 2020

Citation:

Tohyama M (2020) Applications of Time-Dependent Density-Matrix Approach. Front. Phys. 8:67. doi: 10.3389/fphy.2020.00067

\section{Applications of Time-Dependent Density-Matrix Approach}

\author{
Mitsuru Tohyama* \\ Faculty of Medicine, Kyorin University, Tokyo, Japan
}

The equations of motion for reduced density matrices form a coupled chain known as the Bogoliubov-Born-Green-Kirkwood-Yvon (BBGKY) hierarchy. To close the coupled chain at the two-body level, approximations for a three-body density matrix with one-body and two-body density matrices are needed. The time-dependent density-matrix theory (TDDM) assumes that the three-body density matrix is given by the antisymmetrized products of the one-body and two-body density matrices. In this review the truncation schemes of the BBGKY hierarchy beyond TDDM are discussed and a formulation for the study of excited states which is derived from the time-dependent approach is explained. The truncation schemes and the formulation for excited states are applied to the Lipkin model and the Hubbard model to corroborate their validity. Two realistic applications of the TDDM approaches are also presented. One is the dipole and quadrupole excitations of ${ }^{40} \mathrm{Ca}$ and ${ }^{48} \mathrm{Ca}$ and the other the fusion reactions of ${ }^{16} \mathrm{O}+{ }^{16} \mathrm{O}$.

Keywords: extended TDHF, extended RPA, Lipkin model, Hubbard model, giant resonances, fusion

\section{INTRODUCTION}

The time-dependent Hartree-Fock theory (TDHF) is the basis of the mean-field theories such as the Hartree-Fock theory (HF) and the random-phase approximation (RPA): The HF ground state is given as a stationary solution of the TDHF equation and RPA can be formulated as the small amplitude limit of the TDHF equation. HF and RPA have extensively been used as standard theories to study nuclear structure problem [1]. Extensive TDHF simulations have also been performed for heavy-ion collisions $[2,3]$. However, most experimental data suggest that beyondmean field theories are required for a more realistic description of nuclear structure and reactions. In this paper an approach to extend the mean-field theories based on the equations of motion for reduced density matrices is reviewed. The equations of motion for reduced density matrices form a coupled chain known as the Bogoliubov-Born-Green-Kirkwood-Yvon (BBGKY) hierarchy [4] where the time evolution of an $n$-body density matrix depends on $n$-body and $n+1$-body density matrices. The advantage of such a time-dependent density-matrix approach (TDDMA) is that it is directly connected to TDHF in the lowest-level approximation: The truncation of the BBGKY hierarchy at the level of the one-body density matrix by approximating the twobody density matrix with the antisymmetrized product of the one-body density matrices gives TDHF. A beyond TDHF is obtained by the truncation of the BBGKY hierarchy at the two-body level and it needs approximations for the three-body density matrix. A few truncations schemes have been proposed. The simplest truncation scheme is to replace the three-body density matrix with the antisymmetrized products of the one-body and two-body density matrices, neglecting the correlated part of the three-body density matrix [the three-body correlation matrix $\left(C_{3}\right)$ ] $[5,6]$. This truncation scheme has been called the time-dependent density-matrix theory (TDDM). TDDM has been applied to heavy-ion collisions [7-9] and collective excitations [6, 10-12]. A 
simplified TDDM called TDDM $^{\mathrm{P}}$ [13] where two-body correlations are consider only for a pair of time reversed single-particle (s.p.) states have also been employed to simulate heavy-ion collisions $[13,14]$. It was found that TDDM sometimes overestimates ground-state correlations in a solvable model [15], gives unphysical s.p. occupation probabilities in dynamical simulations [16, 17] and causes divergent dynamical behaviors in highly excited and (or) strongly interacting cases [18, 19]. Obviously the problems originate in the neglect of $C_{3}$ and are related to the loss of $N$-representability [20] which refers to the properties of reduced density matrices derived from an $\mathrm{N}$-body total wavefunction and is completely fulfilled only in the case of the untruncated BBGKY hierarchy. To remedy the difficulties of the naive truncation scheme in the description of ground states, an approximation for $C_{3}$ has been proposed based on perturbative consideration [21], where $C_{3}$ is given by the traced products of the correlated part of the two-body density matrix $\left(C_{2}\right)$. This truncation scheme is referred to as TDDM1 hereafter. The correlation matrices $C_{2}$ and $C_{3}$ are also called the two-body and three-body cumulants and the above approximation for $C_{3}$ corresponds to taking the leading-order terms of the three-body cumulant [22, 23]. It has been demonstrated that TDDM1 improves the TDDM results for the ground states of model Hamiltonians [21, 23] and also ${ }^{16} \mathrm{O}[24]$. In the case of the Lipkin model [25], however, TDDM1 was found to overestimate $C_{3}$ in strongly interacting regions. There, another truncation scheme [26] where $C_{3}$ in TDDM1 is divided by a reduction factor was introduced. This truncation scheme is referred to as TDDM2. In this paper three truncation schemes TDDM, TDDM1 and TDDM2 are explained and their applications are presented.

The small amplitude limit of the TDDMA equations gives an extended RPA (ERPA) which is used for the study of excited states, as is the case of RPA which is formulated as the small amplitude limit of TDHF. RPA and ERPA are also formulated by using the equation of motion approach $[27,28]$. ERPA consists of the coupled equations for the one-body and two-body amplitudes and include the effects of ground-state correlations through the fractional occupation probability $n_{\alpha}$ of a s.p. state $\alpha$ and $C_{2}$. ERPA is related to so far proposed beyond-RPA theories. When the coupling to the two-body amplitude is omitted, the ERPA equation for the one-body amplitude is the same as the selfconsistent RPA (SCRPA) $[29,30]$ equation which includes both $n_{\alpha}$ and $C_{2}$. The neglect of $C_{2}$ in the SCRPA equation corresponds to the renormalized RPA (rRPA) [27, 28] which includes the ground-state correlation effect via $n_{\alpha}$. When the HF ground state is assumed, the equations in ERPA are reduced to those in the second RPA (SRPA) [31]. If particle-hole correlations included in the two-body amplitudes were expressed by phonons, ERPA would be connected the particle-vibration coupling or quasiparticle-phonon models [32]. ERPA has been applied to solvable models $[23,33]$. Realistic cases have also been studied in ERPA [34-36]. Main results of the ERPA applications are presented in this paper.

The TDDM truncation scheme has been used for simulations of heavy-ion collisions [7-9] where the TDDM equations are formulated by using the time-dependent s.p. states which obey a TDHF-like equation. The application of such a
TDDM approach to the fusion reactions of ${ }^{16} \mathrm{O}+{ }^{16} \mathrm{O}$ is also presented.

The paper is organized as follows: The equations of motion for the one-body and two-body density matrices formulated by using a time-independent s.p. basis are given in section 2 and the truncation schemes of the BBGKY hierarchy and the formulation of ERPA are discussed. The applications are made to two model Hamiltonians, the Lipkin model [25] and the onedimensional Hubbard model [37] in section 2 and the obtained results are compared with the exact solutions. The ERPA results for the dipole and quadrupole excitations in ${ }^{40} \mathrm{Ca}$ and ${ }^{48} \mathrm{Ca}$ are presented in section 2 as realistic applications of ERPA. The TDDM formulation using a time-dependent s.p. basis and its application to the fusion reactions of ${ }^{16} \mathrm{O}+{ }^{16} \mathrm{O}$ are given in section 3 . Section 4 is devoted to summary and outlook.

\section{FORMULATION IN TIME-INDEPENDENT SINGLE-PARTICLE BASIS}

The TDDMA equations are formulated for an $N$ fermion system described by the Hamiltonian $H$ consisting of a one-body part $t$ (the kinetic energy term) and a two-body interaction $v$

$$
H=\sum_{\alpha \alpha^{\prime}}\left\langle\alpha|t| \alpha^{\prime}\right\rangle a_{\alpha}^{+} a_{\alpha^{\prime}}+\frac{1}{2} \sum_{\alpha \beta \alpha^{\prime} \beta^{\prime}}\left\langle\alpha \beta|v| \alpha^{\prime} \beta^{\prime}\right\rangle a_{\alpha}^{+} a_{\beta}^{+} a_{\beta^{\prime}} a_{\alpha^{\prime}}
$$

where $a_{\alpha}^{+}$and $a_{\alpha}$ are the creation and annihilation operators of a particle at a s.p. state $\alpha$ and the s.p. states are assumed time-independent.

\subsection{Time-Dependent Density-Matrix Theory and Truncation Schemes}

The TDDMA equations given in Tohyama and Schuck [21] are explained below. They consist of the coupled equations of motion for the one-body density matrix (the occupation matrix) $n_{\alpha \alpha^{\prime}}$ and the correlated part of the two-body density matrix $C_{\alpha \beta \alpha^{\prime} \beta^{\prime}}\left(C_{2}\right)$. These matrices are defined as

$$
\begin{aligned}
n_{\alpha \alpha^{\prime}}(t) & =\left\langle\Phi(t)\left|a_{\alpha^{\prime}}^{+} a_{\alpha}\right| \Phi(t)\right\rangle, \\
C_{\alpha \beta \alpha^{\prime} \beta^{\prime}}(t) & =\rho_{\alpha \beta \alpha^{\prime} \beta^{\prime}}(t)-\mathcal{A}\left(n_{\alpha \alpha^{\prime}}(t) n_{\beta \beta^{\prime}}(t)\right),
\end{aligned}
$$

where $|\Phi(t)\rangle$ is the time-dependent total wavefunction $|\Phi(t)\rangle=$ $\exp [-i H t]|\Phi(t=0)\rangle, \rho_{\alpha \beta \alpha^{\prime} \beta^{\prime}}$ is the two-body density matrix $\left(\rho_{\alpha \beta \alpha^{\prime} \beta^{\prime}}(t)=\left\langle\Phi(t)\left|a_{\alpha^{\prime}}^{+} a_{\beta^{\prime}}^{+} a_{\beta} a_{\alpha}\right| \Phi(t)\right\rangle\right)$ and $\mathcal{A}$ is an operator which properly antisymmetrizes $n_{\alpha \alpha^{\prime}} n_{\beta \beta^{\prime}}$ under the exchange of the s.p. indices such as $\alpha \leftrightarrow \beta$ and $\alpha^{\prime} \leftrightarrow \beta^{\prime}$. Units $\hbar=1$ are used hereafter. The equations of motion for $n_{\alpha \alpha^{\prime}}$ and $C_{\alpha \beta \alpha^{\prime} \beta^{\prime}}$ are derived from

$$
\begin{aligned}
i \dot{n}_{\alpha \alpha^{\prime}} & =\left\langle\Phi(t)\left|\left[a_{\alpha^{\prime}}^{+} a_{\alpha}, H\right]\right| \Phi(t)\right\rangle \\
i \dot{\rho}_{\alpha \beta \alpha^{\prime} \beta^{\prime}} & =\left\langle\Phi(t)\left|\left[a_{\alpha^{\prime}}^{+} a_{\beta^{\prime}}^{+} a_{\beta} a_{\alpha}, H\right]\right| \Phi(t)\right\rangle,
\end{aligned}
$$


by evaluating the commutation relations. They are written as

$$
\begin{aligned}
i \dot{n}_{\alpha \alpha^{\prime}} & =\sum_{\lambda}\left(\epsilon_{\alpha \lambda} n_{\lambda \alpha^{\prime}}-n_{\alpha \lambda} \epsilon_{\lambda \alpha^{\prime}}\right) \\
& +\sum_{\lambda_{1} \lambda_{2} \lambda_{3}}\left[\left\langle\alpha \lambda_{1}|v| \lambda_{2} \lambda_{3}\right\rangle C_{\lambda_{2} \lambda_{3} \alpha^{\prime} \lambda_{1}}-C_{\alpha \lambda_{1} \lambda_{2} \lambda_{3}}\left\langle\lambda_{2} \lambda_{3}|v| \alpha^{\prime} \lambda_{1}\right\rangle\right]
\end{aligned}
$$

$$
\begin{aligned}
i \dot{C}_{\alpha \beta \alpha^{\prime} \beta^{\prime}} & =\sum_{\lambda}\left(\epsilon_{\alpha \lambda} C_{\lambda \beta \alpha^{\prime} \beta^{\prime}}+\epsilon_{\beta \lambda} C_{\alpha \lambda \alpha^{\prime} \beta^{\prime}}-\epsilon_{\lambda \alpha^{\prime}} C_{\alpha \beta \lambda \beta^{\prime}}\right. \\
& \left.-\epsilon_{\lambda \beta^{\prime}} C_{\alpha \beta \alpha^{\prime} \lambda}\right)+B_{\alpha \beta \alpha^{\prime} \beta^{\prime}}+P_{\alpha \beta \alpha^{\prime} \beta^{\prime}}+H_{\alpha \beta \alpha^{\prime} \beta^{\prime}}+T_{\alpha \beta \alpha^{\prime} \beta^{\prime}},
\end{aligned}
$$

where $\epsilon_{\alpha \alpha^{\prime}}$ is the s.p. energy including the mean field and is given by

$$
\epsilon_{\alpha \alpha^{\prime}}=\left\langle\alpha|t| \alpha^{\prime}\right\rangle+\sum_{\lambda_{1} \lambda_{2}}\left\langle\alpha \lambda_{1}|v| \alpha^{\prime} \lambda_{2}\right\rangle_{A} n_{\lambda_{2} \lambda_{1}}
$$

Here the subscript $A$ means that the corresponding matrix is antisymmetrized. The term $B_{\alpha \beta \alpha^{\prime} \beta^{\prime}}$ in Equation (6) consists of only the occupation matrices and describes 2 particle (p) -2 hole (h) and $2 \mathrm{~h}-2 \mathrm{p}$ excitations, while $P_{\alpha \beta \alpha^{\prime} \beta^{\prime}}$ and $H_{\alpha \beta \alpha^{\prime} \beta^{\prime}}$ contain $\mathrm{C}_{2}$ and express $\mathrm{p}-\mathrm{p}$ (and $\mathrm{h}-\mathrm{h}$ ) and $\mathrm{p}-\mathrm{h}$ correlations to infinite order, respectively [6]. The $T_{\alpha \beta \alpha^{\prime} \beta^{\prime}}$ term gives the coupling to the three-body correlation matrix $\left(C_{3}\right)$

$$
\begin{aligned}
T_{\alpha \beta \alpha^{\prime} \beta^{\prime}} & =\sum_{\lambda_{1} \lambda_{2} \lambda_{3}}\left[\left\langle\alpha \lambda_{1}|v| \lambda_{2} \lambda_{3}\right\rangle C_{\lambda_{2} \lambda_{3} \beta \alpha^{\prime} \lambda_{1} \beta^{\prime}}\right. \\
& +\left\langle\lambda_{1} \beta|v| \lambda_{2} \lambda_{3}\right\rangle C_{\lambda_{2} \lambda_{3} \alpha \alpha^{\prime} \lambda_{1} \beta^{\prime}} \\
& \left.-\left\langle\lambda_{1} \lambda_{2}|v| \alpha^{\prime} \lambda_{3}\right\rangle C_{\alpha \lambda_{3} \beta \lambda_{1} \lambda_{2} \beta^{\prime}}-\left\langle\lambda_{1} \lambda_{2}|v| \lambda_{3} \beta^{\prime}\right\rangle C_{\alpha \lambda_{3} \beta \lambda_{1} \lambda_{2} \alpha^{\prime}}\right]
\end{aligned}
$$

where $C_{\alpha \beta \gamma \alpha^{\prime} \beta^{\prime} \gamma^{\prime}}\left(C_{3}\right)$ is given by

$$
C_{\alpha \beta \gamma \alpha^{\prime} \beta^{\prime} \gamma^{\prime}}=\left\langle\Phi(t)\left|a_{\alpha^{\prime}}^{+} a_{\beta^{\prime}}^{+} a_{\gamma^{\prime}}^{+} a_{\gamma} a_{\beta} a_{\alpha}\right| \Phi(t)\right\rangle-\mathcal{A}\left(n_{\alpha \alpha^{\prime}} \rho_{\beta \gamma \beta^{\prime} \gamma^{\prime}}\right)(9)
$$

Approximations for $C_{3}$ are needed to close the equations of motion within $n_{\alpha \alpha^{\prime}}$ and $C_{2}$. Three truncation schemes TDDM, TDDM1 and TDDM 2 have been proposed. In TDDM, $C_{3}$ is simply omitted $[5,6]$. In TDDM1, $C_{3}$ is given by [21]

$$
\begin{aligned}
& C_{\mathrm{p}_{1} \mathrm{p}_{2} \mathrm{~h}_{1} \mathrm{p}_{3} \mathrm{p}_{4} \mathrm{~h}_{2}}=\sum_{\mathrm{h}} C_{\mathrm{hh}_{1} \mathrm{p}_{3} \mathrm{p}_{4}} C_{\mathrm{p}_{1} \mathrm{p}_{2} \mathrm{~h}_{2} \mathrm{~h}}, \\
& C_{\mathrm{p}_{1} \mathrm{~h}_{1} \mathrm{~h}_{2} \mathrm{p}_{2} \mathrm{~h}_{3} \mathrm{~h}_{4}}=\sum_{\mathrm{p}} C_{\mathrm{h}_{1} \mathrm{~h}_{2} \mathrm{p}_{2} \mathrm{p}} C_{\mathrm{p}_{1} \mathrm{ph}_{3} \mathrm{~h}_{4}},
\end{aligned}
$$

where $\mathrm{p}$ and $\mathrm{h}$ refer to particle and hole states, respectively. These expressions were derived from perturbative consideration using the Coupled-Cluster-Doubles (CCD)-like ground state wavefunction [38]. In a time-independent density-matrix approach in quantum chemistry, known as the contracted Schrödinger equation [20], Mazziotti [22] has proposed a method for constructing the three-body cumulant $\left(C_{3}\right)$ with $n_{\alpha \alpha^{\prime}}$ and $C_{2}$. Equations (10) and (11) describe the leading-order terms in the three-body cumulant [23]. TDDM2 [26] is the most effective in the large $N$ and strongly interacting limits of the Lipkin model and gives

$$
\begin{aligned}
C_{\mathrm{p}_{1} \mathrm{p}_{2} \mathrm{~h}_{1} \mathrm{p}_{3} \mathrm{p}_{4} \mathrm{~h}_{2}} & =\frac{1}{\mathcal{N}} \sum_{\mathrm{h}} C_{\mathrm{hh}_{1} \mathrm{p}_{3} \mathrm{p}_{4}} C_{\mathrm{p}_{1} \mathrm{p}_{2} \mathrm{~h}_{2} \mathrm{~h}}, \\
C_{\mathrm{p}_{1} \mathrm{~h}_{1} \mathrm{~h}_{2} \mathrm{p}_{2} \mathrm{~h}_{3} \mathrm{~h}_{4}} & =\frac{1}{\mathcal{N}} \sum_{\mathrm{p}} C_{\mathrm{h}_{1} \mathrm{~h}_{2} \mathrm{p}_{2} \mathrm{p}} C_{\mathrm{p}_{1} \mathrm{ph}_{3} \mathrm{~h}_{4}},
\end{aligned}
$$

where $\mathcal{N}$ is

$$
\mathcal{N}=1+\frac{1}{4} \sum_{\mathrm{pp}^{\prime} \mathrm{hh}^{\prime}} C_{\mathrm{pp}^{\prime} \mathrm{hh}^{\prime}} C_{\mathrm{hh}^{\prime} \mathrm{pp}^{\prime}}
$$

The factor $\mathcal{N}$ was introduced to simulate many-body effects which reduce $C_{3}$ in large $N$ systems and (or) strongly interacting regions of the Lipkin model. In the perturbative region where the second term on the right-hand side of Equation (26) is smaller than unity, $\mathcal{N}$ has the meaning of the normalization factor of the total wavefunction.

The conservation of the total energy and total particle number is not affected by the truncation schemes for $C_{3}$ as long as its symmetry and anti-symmetry properties under the exchange of s.p. indices is respected. However, the trace relation between the one-body and two-body density matrices $n_{\alpha \alpha^{\prime}}=\sum_{\lambda} \rho_{\alpha \lambda \alpha^{\prime} \lambda} /(N-1)$ is not conserved when any approximation is made for $C_{3}$. This is an example of the loss of $N$-representability [20]. It was pointed out [21] that the fulfillment of the trace relation is drastically improved by going from TDDM to TDDM1. In an attempt to conserve the trace relation, Cassing and Pfitzner [39] proposed an approximation for $C_{3}$ which also contains quadratic terms of $C_{2}$. However, $C_{3}$ is not uniquely determined only by the requirement of the trace relation conservation. In contrast to TDDM1 their quadratic terms do not have the leading-order terms (Equations 10, 11) of the three-body cumulant $[22,23]$ and the dynamical effect of $C_{3}$ was found small in one-dimensional heavy-ion simulations [39]. $C_{3}$ in Reference [39] is not anti-symmetric under the exchange of s.p. indices, which may violate even the conservation of the trace relation as was pointed out by Gherega et al. [17]. There is another attempt [40] to conserve the trace relation, where the equation motion for $C_{3}$ was solved by truncating the BBGKY hierarchy at the three-body level. However, the application of such an approach was limited to model Hamiltonians [40].

\subsection{Ground-State Calculation}

The ground state in TDDMA is given as a stationary solution of the time-dependent equations (Equations 5,6) which satisfies $\dot{n}_{\alpha \alpha^{\prime}}=0$ and $\dot{C}_{2}=0$. Two methods have been employed to obtain the stationary solution. One is the adiabatic method: Equations (5) and (6) are solved by starting from the HF configuration and gradually increasing the strength of the residual interaction such as $v\left(\vec{r}-\vec{r}^{\prime}\right) \times t / T$. This method is based on the Gell-Mann-Low theorem [41] and has often been used to obtain approximate ground states with various time-dependent functionals [11, 13, 14, 42, 43]. To suppress oscillating components which come from the mixing of excited 
states, $T$ must be much larger than the longest period in the system considered. The other method is a usual iterative gradient method which is useful to obtain a rigorously stationary solution. Since it involves matrix inversion, the application of the gradient method is limited to small systems: The gradient method has been employed to obtain the ground states of the Lipkin model [15] and the oxygen, calcium and tin isotopes [34-36] using several s.p. states around the Fermi level $[34,36]$ or the valence neutron s.p. states [35].

\subsection{Excited-States Calculation}

The formulation for excited states can be derived by either taking the small amplitude limit of the TDDM equations or using the equation of motion approach $[1,27]$. Here the formulation based on the equation of motion approach is presented. Let us consider a generalized RPA operator with one-body and two-body sectors

$$
\mathcal{Q}_{\mu}^{+}=\sum_{\lambda \lambda^{\prime}} x_{\lambda \lambda^{\prime}}^{\mu} a_{\lambda}^{+} a_{\lambda^{\prime}}+\sum_{\lambda_{1} \lambda_{2} \lambda_{1}^{\prime} \lambda_{2}^{\prime}} X_{\lambda_{1} \lambda_{2} \lambda_{1}^{\prime} \lambda_{2}^{\prime}}^{\mu} a_{\lambda_{1}}^{+} a_{\lambda_{2}}^{+} a_{\lambda_{2}^{\prime}} a_{\lambda_{1}^{\prime}},
$$

where there is no restriction on the s.p. indices: They can be both $\mathrm{p}$ and $\mathrm{h}$. As usual with the equation of motion approach, the properties of the excitation operator $\mathcal{Q}_{\mu}^{+}\left|\Phi_{0}\right\rangle=\left|\Phi_{\mu}\right\rangle$ and $\mathcal{Q}_{\mu}\left|\Phi_{0}\right\rangle=0$ are assumed and the following equations of motion satisfied by exact states are taken into account

$$
\begin{aligned}
\left\langle\Phi_{0}\left|\left[a_{\lambda}^{+} a_{\lambda^{\prime}}, H\right]\right| \Phi_{\mu}\right\rangle & \left.=\omega_{\mu} \Phi_{0}\left|a_{\lambda}^{+} a_{\lambda^{\prime}}\right| \Phi_{\mu}\right\rangle \\
\left\langle\Phi_{0}\left|\left[a_{\lambda_{1}}^{+} a_{\lambda_{2}}^{+} a_{\lambda_{2}^{\prime}} a_{\lambda_{1}^{\prime}}, H\right]\right| \Phi_{\mu}\right\rangle & =\omega_{\mu}\left\langle\Phi_{0}\left|a_{\lambda_{1}}^{+} a_{\lambda_{2}}^{+} a_{\lambda_{2}^{\prime}} a_{\lambda_{1}^{\prime}}\right| \Phi_{\mu}\right\rangle,
\end{aligned}
$$

where $\omega_{\mu}$ is the excitation energy of an excited state $\left|\Phi_{\mu}\right\rangle$. The equations for $x_{\lambda \lambda^{\prime}}^{\mu}$ and $X_{\lambda_{1} \lambda_{2} \lambda_{1}^{\prime} \lambda_{2}^{\prime}}^{\mu}$ are obtained by inserting Equation (15) into the above equations. They are written in matrix form $[15,40]$

$$
\left(\begin{array}{ll}
A & B \\
C & D
\end{array}\right)\left(\begin{array}{l}
x^{\mu} \\
X^{\mu}
\end{array}\right)=\omega_{\mu}\left(\begin{array}{ll}
S_{1} & T_{1} \\
T_{2} & S_{2}
\end{array}\right)\left(\begin{array}{l}
x^{\mu} \\
X^{\mu}
\end{array}\right) .
$$

The Hamiltonian matrices $A, B, C$ and $D$ are given by

$$
\begin{aligned}
A\left(\alpha \alpha^{\prime}: \lambda \lambda^{\prime}\right) & =\left\langle\Phi_{0}\left|\left[\left[a_{\alpha^{\prime}}^{+} a_{\alpha}, H\right], a_{\lambda}^{+} a_{\lambda^{\prime}}\right]\right| \Phi_{0}\right\rangle, \\
B\left(\alpha \alpha^{\prime}: \lambda_{1} \lambda_{2} \lambda_{1}^{\prime} \lambda_{2}^{\prime}\right) & =C^{+} \\
& =\left\langle\Phi_{0}\left|\left[\left[a_{\alpha^{\prime}}^{+} a_{\alpha}, H\right], a_{\lambda_{1}}^{+} a_{\lambda_{2}}^{+} a_{\lambda_{2}^{\prime}} a_{\lambda_{1}^{\prime}}\right]\right| \Phi_{0}\right\rangle \\
D\left(\alpha \beta \alpha^{\prime} \beta^{\prime}: \lambda_{1} \lambda_{2} \lambda_{1}^{\prime} \lambda_{2}^{\prime}\right) & =\left\langle\Phi_{0}\left|\left[\left[a_{\alpha^{\prime}}^{+} a_{\beta^{\prime}}^{+} a_{\beta} a_{\alpha}, H\right], a_{\lambda_{1}}^{+} a_{\lambda_{2}}^{+} a_{\lambda_{2}^{\prime}} a_{\lambda_{1}^{\prime}}\right]\right| \Phi_{0}\right\rangle .
\end{aligned}
$$

The norm matrices $S_{1}, T_{1}, T_{2}$, and $S_{2}$ are given as

$$
\begin{aligned}
S_{1}\left(\alpha \alpha^{\prime}: \lambda \lambda^{\prime}\right) & =\left\langle\Phi_{0}\left|\left[a_{\alpha^{\prime}}^{+} a_{\alpha}, a_{\lambda}^{+} a_{\lambda^{\prime}}\right]\right| \Phi_{0}\right\rangle, \\
T_{1}\left(\alpha \alpha^{\prime}: \lambda_{1} \lambda_{2} \lambda_{1}^{\prime} \lambda_{2}^{\prime}\right) & =T_{2}^{+}=\left\langle\Phi_{0}\left|\left[a_{\alpha^{\prime}}^{+} a_{\alpha}, a_{\lambda_{1}}^{+} a_{\lambda_{2}}^{+} a_{\lambda_{2}^{\prime}} a_{\lambda_{1}^{\prime}}\right]\right| \Phi_{0}\right\rangle,
\end{aligned}
$$

$S_{2}\left(\alpha \beta \alpha^{\prime} \beta^{\prime}: \lambda_{1} \lambda_{2} \lambda_{1}^{\prime} \lambda_{2}^{\prime}\right)=\left\langle\Phi_{0}\left|\left[a_{\alpha^{\prime}}^{+} a_{\beta^{\prime}}^{+} a_{\beta} a_{\alpha}, a_{\lambda_{1}}^{+} a_{\lambda_{2}}^{+} a_{\lambda_{2}^{\prime}} a_{\lambda_{1}^{\prime}}\right]\right| \Phi_{0}\right\rangle$.

These matrices are evaluated by assuming $\left|\Phi_{0}\right\rangle$ to be the ground state in TDDMA. This means that the effects of ground-state correlations are included in the above matrices through $n_{\alpha \alpha^{\prime}}$ and $C_{2}$. All matrix elements in Equation (18) are given in Tohyama and Schuck [40]. The one-body sector of Equation (18), $A x^{\mu}=$ $\omega_{\mu} S_{1} x^{\mu}$, is explicitly shown below to explain how $n_{\alpha \alpha^{\prime}}$ and $C_{2}$ are included. The matrix $S_{1}$ is given by

$$
S_{1}\left(\alpha \alpha^{\prime}: \lambda \lambda^{\prime}\right)=\left(n_{\alpha^{\prime} \alpha^{\prime}}-n_{\alpha \alpha}\right) \delta_{\alpha \lambda} \delta_{\alpha^{\prime} \lambda^{\prime}}
$$

and $A$ by

$$
\begin{aligned}
A\left(\alpha \alpha^{\prime}: \lambda \lambda^{\prime}\right) & =\left(\epsilon_{\alpha}-\epsilon_{\alpha^{\prime}}\right)\left(n_{\alpha^{\prime} \alpha^{\prime}}-n_{\alpha \alpha}\right) \delta_{\alpha \lambda} \delta_{\alpha^{\prime} \lambda^{\prime}} \\
& +\left(n_{\alpha^{\prime} \alpha^{\prime}}-n_{\alpha \alpha}\right)\left(n_{\lambda^{\prime} \lambda^{\prime}}-n_{\lambda \lambda}\right)\left\langle\alpha \lambda^{\prime}|v| \alpha^{\prime} \lambda\right\rangle_{A} \\
& -\delta_{\alpha^{\prime} \lambda^{\prime}} \sum_{\gamma \gamma^{\prime} \gamma^{\prime \prime}}\left\langle\alpha \gamma|v| \gamma^{\prime} \gamma^{\prime \prime}\right\rangle C_{\gamma^{\prime} \gamma^{\prime \prime} \lambda \gamma} \\
& -\delta_{\alpha \lambda} \sum_{\gamma \gamma^{\prime} \gamma^{\prime \prime}}\left\langle\gamma \gamma^{\prime}|v| \alpha^{\prime} \gamma^{\prime \prime}\right\rangle C_{\lambda^{\prime} \gamma^{\prime \prime} \gamma \gamma^{\prime}} \\
& +\sum_{\gamma \gamma^{\prime}}\left(\left\langle\alpha \gamma|v| \lambda \gamma^{\prime}\right\rangle_{A} C_{\lambda^{\prime} \gamma^{\prime} \alpha^{\prime} \gamma}+\left\langle\lambda^{\prime} \gamma|v| \alpha^{\prime} \gamma^{\prime}\right\rangle_{A} C_{\alpha \gamma^{\prime} \lambda \gamma}\right) \\
& -\sum_{\gamma \gamma^{\prime}}\left(\left\langle\alpha \lambda^{\prime}|v| \gamma \gamma^{\prime}\right\rangle C_{\gamma \gamma^{\prime} \alpha^{\prime} \lambda}+\left\langle\gamma \gamma^{\prime}|v| \alpha^{\prime} \lambda\right\rangle C_{\alpha \lambda^{\prime} \gamma \gamma^{\prime}}\right),
\end{aligned}
$$

where $\epsilon_{\alpha \alpha^{\prime}}$ and $n_{\alpha \alpha^{\prime}}$ are assumed to be diagonal for simplicity. The first two terms on the right-hand side of Equation (22) are of the same form as the RPA and rRPA euqations, the next two terms with $C_{2}$ and the Kronecker delta $\delta_{\alpha \alpha^{\prime}}$ describe the self-energies of the $\alpha-\alpha^{\prime}$ configurations due to ground-state correlations $[29,44]$, and the other terms with $C_{2}$ are interpreted as the vertex corrections [29, 44]. Equation (18) has the most general form of beyond RPA theories: It is reduced to SRPA when the ground-state correlations are neglected and the onebody sector of Equation (18) $A x^{\mu}=\omega_{\mu} S_{1} x^{\mu}$ is formally the same as the equation in SCRPA. Equation (18) is referred to as the extended RPA (ERPA) hereafter.

Although ERPA has great advantages over other extended RPA theories, it is worth pointing out its limitations. The numbers of the matrix elements of $C_{2}$ and $X_{\alpha \beta \alpha^{\prime} \beta^{\prime}}^{\mu}$ increase rapidly with increasing number of the s.p. states. Therefore, truncation of the s.p. space is required in realistic applications. As shown below, basic effects of two-body correlations can be described with rather small s.p. space, however. The other limitation is concerned with hermiticity of $D$ in Equation (18), which is related to the truncation of the BBGKY hierarchy. Equation (21) contains $C_{3}$ and it is approximated depending on the truncation scheme. Hermiticity of $D$ which is guaranteed only when all the matrix elements of $C_{3}$ satisfy the stationary condition as those of $n_{\alpha \alpha^{\prime}}$ and $C_{2}$ do is not fulfilled [40] when any approximated is made for $C_{3}$. The non-hermiticity has not caused serious problems in the applications thus far considered, though. In the case of the Lipkin model, an attempt [40] to obtain Hermitian D was carried out by solving the equation of motion for $C_{3}$.

\subsection{Applications}

TDDMA's in the time-independent s.p. basis have been applied to model Hamiltonians $[23,33]$ to corroborate their validity: 
The Lipkin model [25] was used to compare three truncation schemes TDDM, TDDM1 and TDDM2. Comparison of ERPA with other beyond-RPA theories, rRPA, SCRPA and SRPA was performed for the one-dimensional Hubbard model [37]. As realistic applications of ERPA, the quadrupole excitations of oxygen isotopes [34], the low-lying quadrupole states in tin isotopes [35] and the dipole and quadrupole excitations of ${ }^{40} \mathrm{Ca}$ and ${ }^{48} \mathrm{Ca}$ [36] have been studied. In the following some of the results are presented.

\subsubsection{Lipkin Model}

The Lipkin model [25] has extensively been used to test theoretical models. It describes an $\mathrm{N}$-fermions system with two $N$-fold degenerate levels. The upper (lower) levels have energies $\epsilon / 2(-\epsilon / 2)$ and quantum number $p(-p)$ with $p=1,2, \ldots, N$. The Hamiltonian is given by

$$
H=\epsilon J_{z}+\frac{V}{2}\left(J_{+}^{2}+J_{-}^{2}\right),
$$

where the operators are the followings

$$
\begin{aligned}
& J_{z}=\frac{1}{2} \sum_{p=1}^{N}\left(c_{p}^{+} c_{p}-c_{-p}{ }^{+} c_{-p}\right), \\
& J_{+}=J_{-}^{+}=\sum_{p=1}^{N} c_{p}^{+} c_{-p .}
\end{aligned}
$$

For $\chi=|V|(N-1) / \epsilon \leq 1$ the HF ground state is given by $|\mathrm{HF}\rangle=\prod_{p=1}^{N} c_{-p}^{+}|0\rangle$, where $|0\rangle$ is the true vacuum. For $\chi>1$ the lowest s.p. states are obtained by the transformation

$$
\left(\begin{array}{c}
a_{-p}^{+} \\
a_{p}^{+}
\end{array}\right)=\left(\begin{array}{cc}
\cos \alpha & \sin \alpha \\
-\sin \alpha & \cos \alpha
\end{array}\right)\left(\begin{array}{c}
c_{-p}^{+} \\
c_{p}^{+}
\end{array}\right)
$$

where $\alpha$ satisfies $\cos 2 \alpha=1 / \chi$. The HF ground state in this case is often called the "deformed" HF (DHF) state and is given by $|\operatorname{DHF}(\alpha)\rangle=\prod_{p=1}^{N} a_{-p}^{+}|0\rangle$.

The truncation schemes TDDM, TDDM1 and TDDM2 have been applied to the Lipkin model and it was found that the simplest scheme TDDM gives the exact solutions in the limits of large $N$ and $\chi$ [45]. This is due to the unique property of the Lipkin model that the ground-state energy in DHF becomes exact in such limits [1]. The relation between the density-matrices in DHF and TDDM is discussed below. The occupation matrix in DHF is given by $n_{-p-p}=\cos ^{2} \alpha, n_{p p}=1-n_{-p-p}=$ $\sin ^{2} \alpha$, and $n_{p-p}=\cos \alpha \sin \alpha$. The two-body and three-body density matrices in DHF are given by the above elements of the occupation matrix. For example the $2 \mathrm{p}-2 \mathrm{~h}$ and $\mathrm{ph}$-ph elements of the two-body density matrix are expressed as

$$
\begin{aligned}
\rho_{p p^{\prime}-p-p^{\prime}} & =\left\langle\operatorname{DHF}(\alpha)\left|c_{-p}^{+} c_{-p^{\prime}}^{+} c_{p^{\prime}} c_{p}\right| \operatorname{DHF}(\alpha)\right\rangle \\
& =\cos ^{2} \alpha \sin ^{2} \alpha=n_{p-p} n_{p^{\prime}-p^{\prime}}=\rho_{p-p^{\prime}-p p^{\prime}}\left(p \neq p^{\prime}\right) .
\end{aligned}
$$

Similarly, the three-body density matrix is given by

$$
\rho_{p-p^{\prime} p^{\prime \prime} p p^{\prime}-p^{\prime \prime}}=\cos ^{2} \alpha \sin ^{4} \alpha=n_{p p} \rho_{-p^{\prime} p^{\prime \prime} p^{\prime}-p^{\prime \prime}} .
$$

This means that the correlated part $\left(C_{p-p^{\prime} p^{\prime \prime} p p^{\prime}-p^{\prime \prime}}\right)$ of the threebody density matrix vanishes in DHF. The "spherical" total wavefunction $|\Psi\rangle$ in DHF which does not have the mixing of the $p$ and $-p$ states is given by the two DHF solutions as

$$
|\Psi\rangle=\frac{1}{\sqrt{2}}(|\operatorname{DHF}(\alpha)\rangle+|\operatorname{DHF}(-\alpha)\rangle) .
$$

Since the overlap between $|\operatorname{DHF}(\alpha)\rangle$ and $|\operatorname{DHF}(-\alpha)\rangle$ is negligibly in the large $\chi$ and $N$ limits, the three-body density matrix calculated with $|\Psi\rangle$ has also no correlated part. This is the reason why the results in TDDM approach the exact solutions in the large $\chi$ and $N$ limits.

The ground-state energy $E_{0}$ calculated in TDDM (solid line) for $N=12$ and 50 is presented respectively in Figures 1, 2 as a function of $\chi$. The dashed and green (gray) lines denote the results in TDDM1 and TDDM2, respectively. In the case of $N=50$ the results in TDDM2 are not displayed because they lie between the TDDM results and the exact values. The dotted and dot-dashed lines depict the results in HF and DHF $(\chi>1)$ and the exact values, respectively. As seen in Figures 1, 2, the factor $\mathcal{N}$ in Equations (12) and (13) plays a role in greatly reducing $C_{3}$, making TDDM2 almost equivalent to TDDM for $N=50$. In the limits of large $N$ and $\chi$ both TDDM and DHF results become close to the exact solutions. In the transitional region $\chi \approx 1$, however, TDDM1 and TDDM2 were found better than TDDM and DHF [45]. In the case of $N=12$ this extends to $\chi \approx 2$ as seen in Figure 1.

The excitation energies of the first and second excited states calculated from the small oscillations of the TDDM solutions (dots) are compared with the exact solutions (dot-dashed line) in Figures 3, 4 for $N=200$ where TDDM is supposed to give the nearly exact ground state. The dotted lines in Figures 3, 4 depict the results in RPA and the "deformed" RPA $(\chi>1)$ for the first excited state. In contrast to RPA and the deformed RPA TDDM reproduces the smoothly decreasing excitation energy of the first excited state with increasing interaction strength beyond $\chi=1$. Figure 4 shows that TDDM also gives good description of the second excited state. As seen in Figure 4, the excitation energies for the first excited state calculated in the deformed RPA become close to the exact values for the second excited state [1] with increasing $\chi$. The excited states were also calculated for a small system with $N=4$ by using the ground states in TDDM [15] and TDDM1 [23], and it was found that the TDDM1 ground state gives much better results.

As was pointed out above, the fact that TDDM becomes exact in large $N$ and $\chi$ limits is due to the unique feature of the Lipkin model that the mean-field theory DHF gives the exact solutions in such limits. In the transitional region $\chi \approx$ 1 TDDM1 and TDDM2 give better description of the exact solutions than TDDM, and the applications to the ground states of other solvable models $[23,26]$ and ${ }^{16} \mathrm{O}[24]$ also showed that TDDM1 largely improves TDDM whereas the improvement 


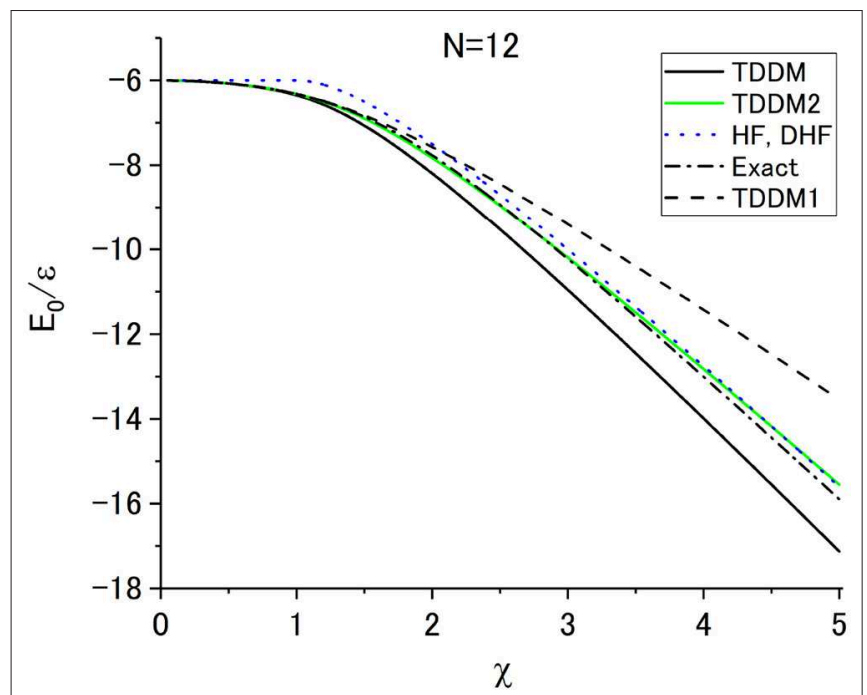

FIGURE 1 | Ground-state energy in TDDM (solid line) as a function of $\chi=|V|(N-1) / \epsilon$ for $N=12$. The results in TDDM1 where the three-body correlation matrix is given by Equations (10) and (11) are shown with the dashed line. The green (gray) line depicts the results in TDDM2 where the three-body correlation matrix is given by Equations (12) and (13). The results in $\mathrm{HF}$ and $\operatorname{DHF}(\chi>1)$ are depicted with the dotted line. The exact values are given by the dot-dashed line. Adapted from Tohyama and Schuck [45] with permission from Società Italiana di Fisica/Springer-Verlag GmbH Germany.

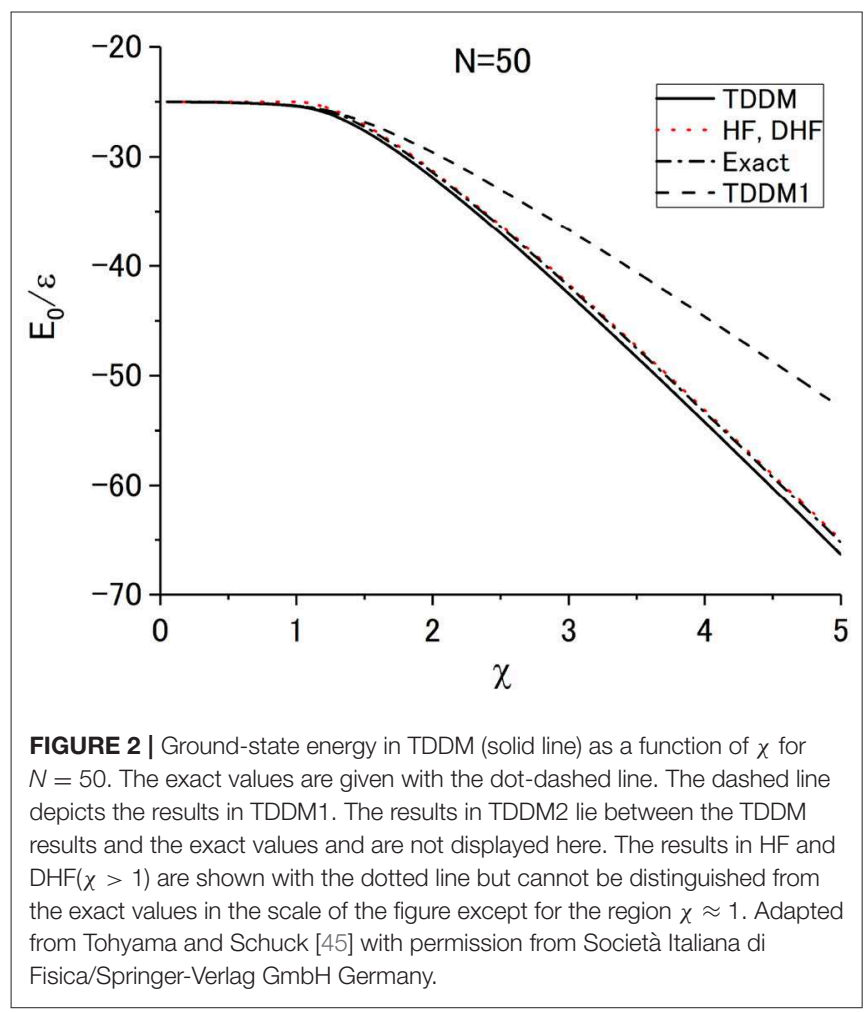

from TDDM1 to TDDM2 is not large [26]. Therefore, TDDM1 or TDDM2 may be a useful truncation scheme to be applied to realistic cases except for strongly interacting regions.

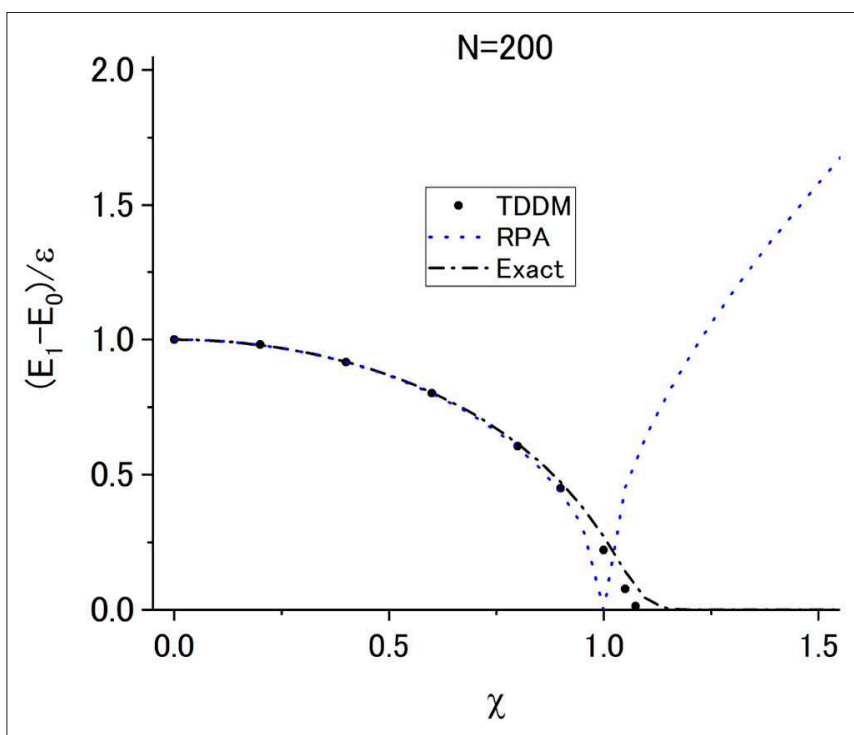

FIGURE 3 | Excitation energy of the first excited state calculated in TDDM (dots) as a function of $\chi$ for $N=200$. The exact values are shown with the dot-dashed line. The dotted line depicts the results in RPA. Adapted from Tohyama and Schuck [45] with permission from Società Italiana di Fisica/Springer-Verlag GmbH Germany.

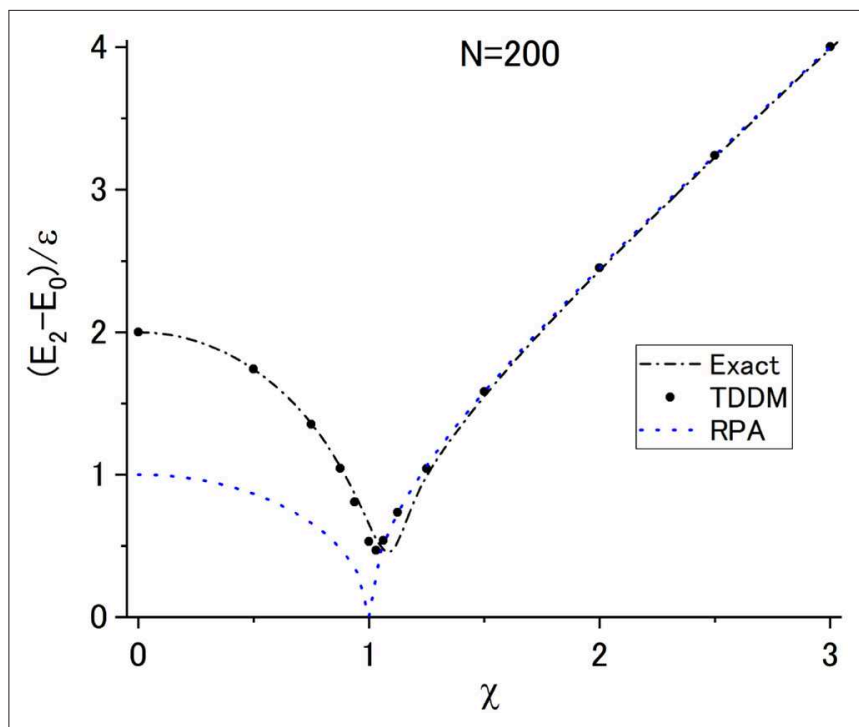

FIGURE 4 | Same as Figure 3 but for the second excited state. The dotted line depicts the RPA results for the first excited state. Adapted from Tohyama and Schuck [45] with permission from Società Italiana di Fisica/Springer-Verlag GmbH Germany.

\subsubsection{One-Dimensional Hubbard Model}

ERPA based on the TDDM1 ground state has been applied to the one-dimensional (1-D) Hubbard model [37] to compare with other beyond RPA theories [33]. The Hubbard model is one of the most widespread models to investigate strong electron correlations and has often been used to corroborate the validity of beyond RPA theories [46]. In momentum space the Hamiltonian 


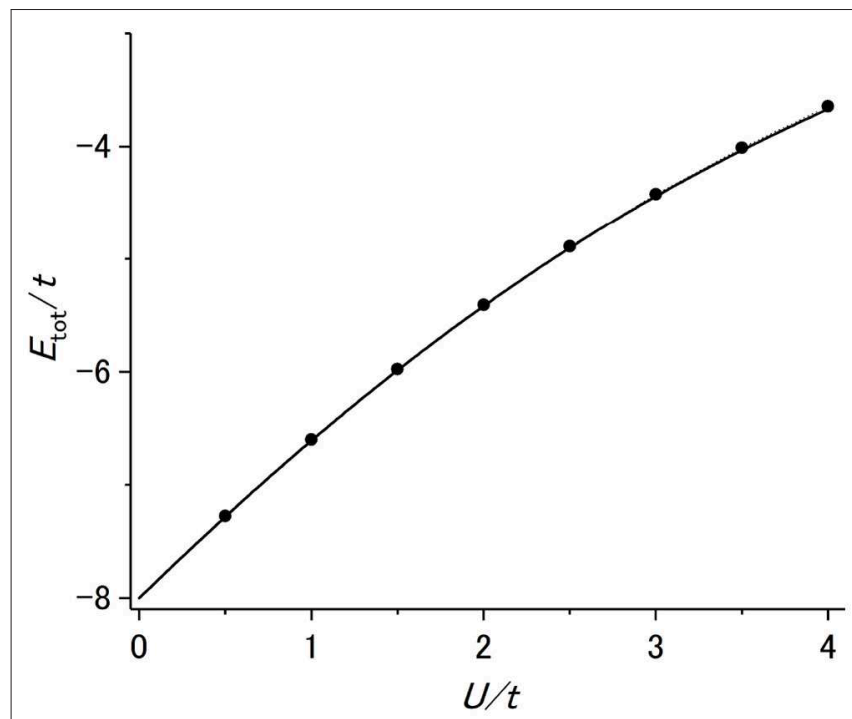

FIGURE 5 | Ground-state energy $E_{\text {tot }}$ calculated in TDDM1 (dots) as a function of $U / t$ for $N=6$ with half-filling. The exact solutions are displayed with the solid line. Adapted from Tohyama [33] under the Creative Commons CCBY license.

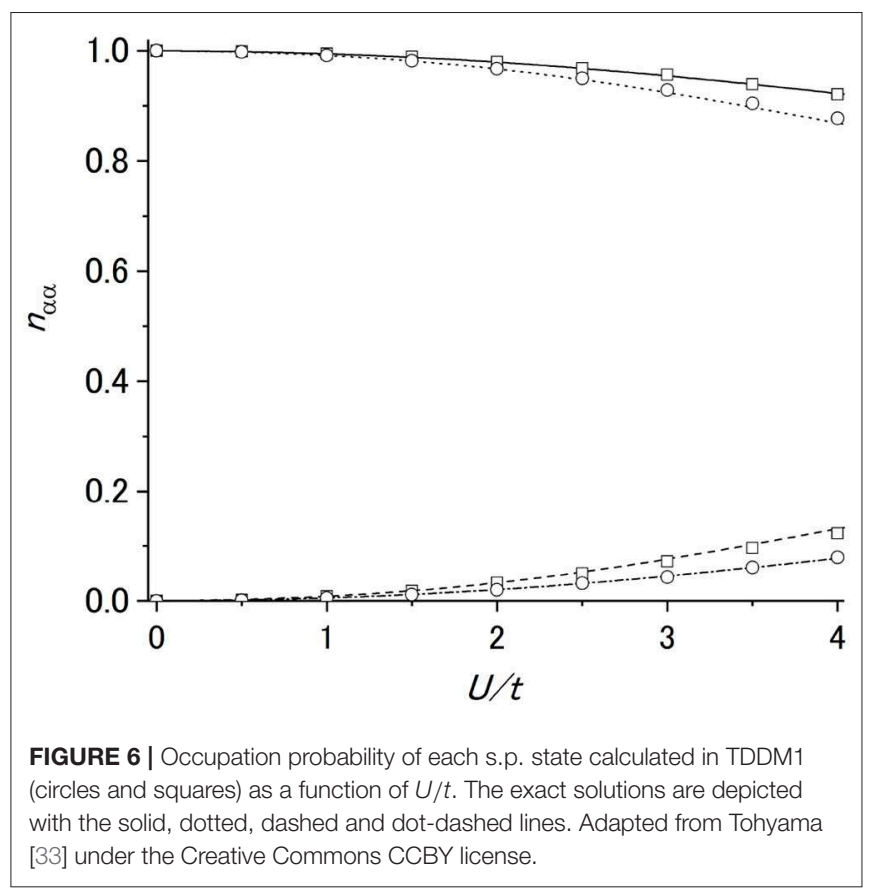

excited mainly by the one-body operator $a_{k_{4} \uparrow}^{+} a_{k_{2} \uparrow}-a_{k_{4} \downarrow}^{+} a_{k_{2} \downarrow}$. Since the s.p. states are partially occupied, the $h-h$ and $p-p$ transitions such as $k_{1} \rightarrow k_{2}$ and $k_{6} \rightarrow k_{5}$ also contribute in rRPA, SCRPA and ERPA. In Figure 7 the excitation energies in ERPA (dots), RPA (open triangles), rRPA (filled triangles), SCRPA (squares) and SRPA (crosses) are shown as functions of $U / t$. The exact solutions are given with the solid line. The rRPA and SCRPA results are calculated with $n_{\alpha \alpha}$ and $C_{\alpha \beta \alpha^{\prime} \beta^{\prime}}$ which are not self-consistently determined by the $\mathrm{p}-\mathrm{h}$ and $\mathrm{h}-\mathrm{p}$ amplitudes $[28,29]$ but given by the TDDM1 calculations. In the case of a repulsive interaction, the excitation energy of a spin mode where the s.p. transitions between spin-up states and spin-down states destructively interfere decreases with increasing $U$. The results in RPA agree rather well with the exact solutions. In rRPA there are two states below $E / t<2$. The main components of the lower state at $E / t \approx 1$ are the $\mathrm{p}-\mathrm{p}$ and $\mathrm{h}-\mathrm{h}$ transitions and the higher state consists of the $\mathrm{p}-\mathrm{h}$ and $\mathrm{h}-\mathrm{p}$ components. Thus in rRPA the configurations consisting of the $\mathrm{p}-\mathrm{p}$ and $\mathrm{h}-\mathrm{h}$ components appear as the lowest state as if it is a physical state. This indicates that it is not appropriate to include the ground-state correlation effects only through $n_{\alpha}$. In SCRPA the states originating from the $\mathrm{p}-\mathrm{p}$ and $\mathrm{h}-\mathrm{h}$ transitions gain self energies and move to the high energy region $(E / t>10)$. This is because the terms in Equation (26) with $C_{2}$ are divided by the small values $n_{\mathrm{pp}}-n_{\mathrm{p}^{\prime} \mathrm{p}^{\prime}}$ or $n_{\mathrm{hh}}-n_{\mathrm{h}^{\prime} \mathrm{h}^{\prime}}$ when $A x^{\mu}=S_{1} x^{\mu}$ is solved. Thus in SCRPA the states consisting of the $\mathrm{p}-\mathrm{p}$ and $\mathrm{h}-\mathrm{h}$ components are energetically separated from the lowest state. The excitation energies of the lowest state calculated in SCRPA, however, exceed significantly the exact values. This is due to the neglect of the coupling to the two-body amplitudes. SRPA includes the coupling to the twobody amplitudes though the ground-state correlation effects are neglected. As shown in Figure 7 with the crosses, the coupling to 


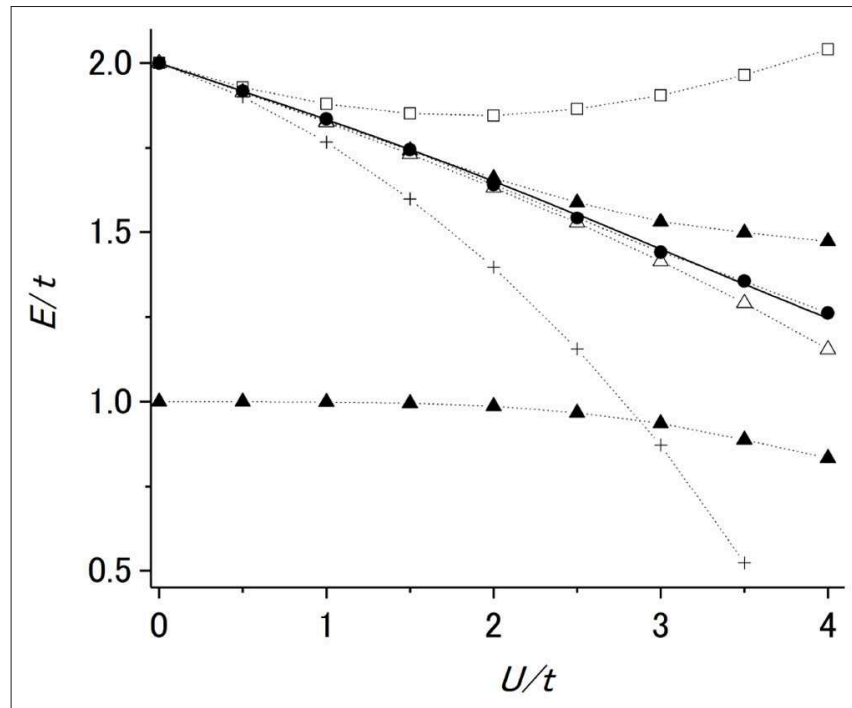

FIGURE 7 | Excitation energy of the spin mode with momentum transfer $q=\pi / 3$ as a function of $U / t$ calculated in ERPA (dots). The results in RPA, rRPA, SCRPA, and SRPA are denoted with the open triangles, filled triangles, squares and crosses, respectively. The exact solutions are depicted with the solid line. Adapted from Tohyama [33] under the Creative Commons CCBY license.

the $2 \mathrm{p}-2 \mathrm{~h}$ amplitudes considered in SRPA downwardly shift the RPA results with increasing $U$ and SRPA collapses at $U / t=3.9$. The results in ERPA have reasonable agreement with the exact solutions. The coupling to the two-body amplitudes included in ERPA plays a role in bringing down the results in SCRPA.

From the application to the 1-D Hubbard model it was clarified that when the couping to the two-body amplitude is considered, the effects of ground-state correlations should also be included, and vice versa. Therefore, rRPA, SCRPA and SRPA which only include either the ground-state correlations effects or the coupling to the two-body amplitude cannot give satisfactory results.

\subsubsection{Dipole and Quadrupole Excitations of ${ }^{40} \mathrm{Ca}$ and ${ }^{48} \mathrm{Ca}$}

In this subsection the applications of ERPA to the dipole excitation of ${ }^{48} \mathrm{Ca}$ and the quadrupole excitation of ${ }^{40} \mathrm{Ca}$ [36] are presented. It is demonstrated that the effects of ground-state correlations which are not fully incorporated in other beyond RPA theories play a significant role in the fragmentation of transition strengths.

Since the numbers of $C_{2}$ and $X_{\alpha \beta \alpha^{\prime} \beta^{\prime}}^{\mu}$ increase rapidly with the number of the s.p. states, rather sever truncation of the s.p. space is required in realistic applications. The occupation probability $n_{\alpha \alpha}$ and $C_{2}$ were calculated within TDDM by using the truncated s.p. basis consisting of the $2 s-1 d$ and $1 f-2 p$ states, and only the $2 \mathrm{p}-2 \mathrm{~h}$ and $2 \mathrm{~h}-2 \mathrm{p}$ elements of $C_{2}$ were included to reduce the dimension size. It was pointed out in Reference [24] that TDDM with this truncation of $C_{2}$ gives as good results for the ground state of ${ }^{16} \mathrm{O}$ as TDDM1 with all components of $C_{2}$. The Skyrme III force [47] was used to obtain the s.p.
TABLE 1 | Single-particle energies $\epsilon_{\alpha}$ and occupation probabilities $n_{\alpha \alpha}$ calculated in TDDM for ${ }^{40} \mathrm{Ca}$.

\begin{tabular}{lccccc}
\hline & \multicolumn{2}{c}{$\boldsymbol{\epsilon}_{\alpha}[\mathrm{MeV}]$} & & \multicolumn{3}{c}{$\boldsymbol{n}_{\alpha \alpha}$} \\
\cline { 2 - 3 } \cline { 5 - 6 } Orbit & Proton & Neutron & & Proton & Neutron \\
\hline $1 d_{5 / 2}$ & -15.6 & -22.9 & & 0.923 & 0.924 \\
$1 d_{3 / 2}$ & -9.4 & -16.5 & & 0.884 & 0.884 \\
& & & & $(0.65 \pm 0.05)$ & $(0.80 \pm 0.11)$ \\
$2 s_{1 / 2}$ & -8.5 & -15.9 & & 0.846 & 0.846 \\
$1 f_{7 / 2}$ & -3.4 & -10.4 & & 0.154 & 0.154 \\
\hline
\end{tabular}

Observed occupation probabilities [48] are shown for the $1 d_{3 / 2}$ states.

wavefunctions which satisfy a HF-like $n_{\alpha \alpha}$-dependent equation. In Pfitzner et al. [11] and Peter et al. [12] a fixed harmonic oscillator basis was chosen to facilitate the calculations of twobody matrix elements when the TDDM equations were applied to the study of giant resonances. A simplified interaction which contains only the $t_{0}$ and $t_{3}$ terms of the Skyrme III force was used as the residual interaction. The ground states were calculated with the iterative gradient method [15]. The one-body amplitudes $x_{\alpha \alpha^{\prime}}^{\mu}$ in Equation (18) were defined with a large number of s.p. states including those in the continuum to satisfy the energy-weighted sum rule: The continuum states were discretized by confining the wavefunctions in a sphere with radius $15 \mathrm{fm}$ and all the s.p. states with $\epsilon_{\alpha} \leq 50 \mathrm{MeV}$ and $j_{\alpha} \leq 11 / 2 \hbar$ were included. The residual interaction in Equation (18) was assumed to have the same form as that used in the ground-state calculations. Since the residual interaction differs from the effective interaction used in the calculation of the s.p. states, it is necessary to reduce the strength of the residual interaction. The reduction factors 0.66 and 0.69 for ${ }^{40} \mathrm{Ca}$ and ${ }^{48} \mathrm{Ca}$, respectively, were determined so that the spurious mode corresponding to the center-of-mass motion has zero excitation energy in RPA. To reduce the number of the two-body amplitudes, only the $2 \mathrm{p}-2 \mathrm{~h}$ and $2 \mathrm{~h}-2 \mathrm{p}$ components of $X_{\alpha \beta \alpha^{\prime} \beta^{\prime}}^{\mu}$ were considered for the $2 s-1 d$ and $2 p-1 f$ states.

The occupation probabilities calculated in TDDM for ${ }^{40} \mathrm{Ca}$ and ${ }^{48} \mathrm{Ca}$ are given in Tables $\mathbf{1}, \mathbf{2}$, respectively. They deviate more than $10 \%$ from the HF values $\left(n_{\alpha \alpha}=1\right.$ or 0$)$ in ${ }^{40} \mathrm{Ca}$. This indicates that the ground state of ${ }^{40} \mathrm{Ca}$ is highly correlated as an RPA study [49] and perturbative calculations [31, 50] have already shown. Since the occupation of the neutron $1 f_{7 / 2}$ state in ${ }^{48} \mathrm{Ca}$ blocks some $2 \mathrm{p}-2 \mathrm{~h}$ excitations, the ground-state correlations are weaker in ${ }^{48} \mathrm{Ca}$ than in ${ }^{40} \mathrm{Ca}$. As will be discussed below, the fractional occupation of the $2 p-1 f$ states plays an important role in the fragmentation of dipole and quadrupole transition strengths. Occupation probabilities deduced from ground-state-to-groundstate $(p, d)$ and $\left(e, e^{\prime} p\right)$ reactions [48] are also shown for some s.p. states in Tables 1, 2 (values in parentheses). These values also strongly deviate from the $\operatorname{HF}$ value $\left(n_{\alpha}=1\right)$. The TDDM results cannot be directly compared with these data, however. A more appropriate formalism such as the odd-particle number RPA [51] which deals with odd particle systems is needed to compare with experiment.

The strength function for the isovector dipole excitation in ${ }^{48} \mathrm{Ca}$ calculated in ERPA (solid line) is displayed in Figure 8. 
TABLE 2 | Same as Table 1 but for ${ }^{48} \mathrm{Ca}$.

\begin{tabular}{lccccc}
\hline & \multicolumn{2}{c}{$\epsilon_{\alpha}[\mathrm{MeV}]$} & & \multicolumn{2}{c}{$\boldsymbol{n}_{\alpha \alpha}$} \\
\cline { 2 - 3 } \cline { 5 - 6 } Orbit & Proton & Neutron & & Proton & Neutron \\
\hline $1 d_{5 / 2}$ & -22.6 & -22.4 & & 0.963 & 0.965 \\
$1 d_{3 / 2}$ & -17.1 & -17.0 & & 0.952 & 0.940 \\
$2 s_{1 / 2}$ & -15.1 & -16.4 & & 0.905 & 0.932 \\
& & & & $(0.54 \pm 0.04)$ & \\
$1 f_{7 / 2}$ & -10.6 & -10.6 & & 0.059 & 0.919 \\
& & & & & $0.73 \pm 0.14)$ \\
$2 p_{3 / 2}$ & -1.7 & -3.8 & & - & 0.103 \\
$2 p_{1 / 2}$ & 0.1 & -2.0 & & - & 0.064 \\
$1 f_{5 / 2}$ & -2.2 & -1.9 & & 0.022 & 0.116 \\
\hline
\end{tabular}

Observed occupation probabilities [48] are shown for the proton $2 s_{1 / 2}$ and neutron $1 f_{7 / 2}$ states.

The dotted and dot-dashed lines depict the results in RPA and SRPA, respectively. An artificial width $\Gamma=0.5 \mathrm{MeV}$ is used to smooth the distributions. The strength distributions in Figure 8 exhaust about $90 \%$ of the energy-weighted-sum-rule (EWSR) value including the enhancement term given by the $t_{1}$ and $t_{2}$ parameters of the Skyrme III force. A better treatment of the residual interaction and the continuum states is required to fulfill the EWSR value. The sharp peak in RPA corresponds to the giant dipole resonance (GDR). GDR strongly couples to the $2 \mathrm{p}-2 \mathrm{~h}$ states and it is damped both in SRPA and ERPA. The occupation of the neutron $1 f_{7 / 2}$ state in ${ }^{48} \mathrm{Ca}$ allows the $2 \mathrm{p}-2 \mathrm{~h}$ states which include the neutron $1 f_{7 / 2}$ state as a hole state. Since these states have energies close to the energy of GDR, GDR is strongly damped due to the coupling to the $2 \mathrm{p}-2 \mathrm{~h}$ states. The SRPA result in Figure 8 dose not show a strong downward shift of the dipole strength which has been reported in large scale SRPA calculations [53]. This is due to fact that a rather small number of the s.p. states are used to define the $2 \mathrm{p}-2 \mathrm{~h}$ amplitudes. The peak position and width of GDR in ERPA are comparable with the experimental photo absorption cross section [52] as shown in the inset of Figure 8.

ERPA gives 7 states below $10 \mathrm{MeV}$, which are compared with experiment [54] in Figure 9. These states involve the transitions from the partially occupied neutron $2 p_{1 / 2}, 2 p_{3 / 2}$ and $1 f_{5 / 2}$ states and the $\mathrm{p}-\mathrm{p}$ transition components exhaust $15-39 \%$ of the transition amplitude $\left(x^{\mu}, X^{\mu}\right)$. The summed strength below $10 \mathrm{MeV}$ is $213 \times 10^{-3} e^{2} \mathrm{fm}^{2}$, which somewhat overestimates the experimental value $61.5 \pm 7.8 \times 10^{-3} e^{2} \mathrm{fm}^{2}$. SRPA gives two dipole states at $9.2 \mathrm{MeV}$ and $9.3 \mathrm{MeV}$ with the summed strength $21 \times 10^{-3} e^{2} \mathrm{fm}^{2}$. The study of low-lying dipole strength distribution has been attracting strong interests and various theoretical approaches such as the large scale SRPA $[55,56]$ and the quasi-particle phonon coupling models $[57,58]$ have been successfully applied to calcium isotopes.

The strength function for the isoscalar quadrupole excitation in ${ }^{40} \mathrm{Ca}$ calculated in ERPA (solid line) is shown in Figure 10. The dotted and dot-dashed lines depict the results in RPA and SRPA, respectively. The distributions are smoothed with an artificial width $\Gamma=0.5 \mathrm{MeV}$. The energy-weighted sums of
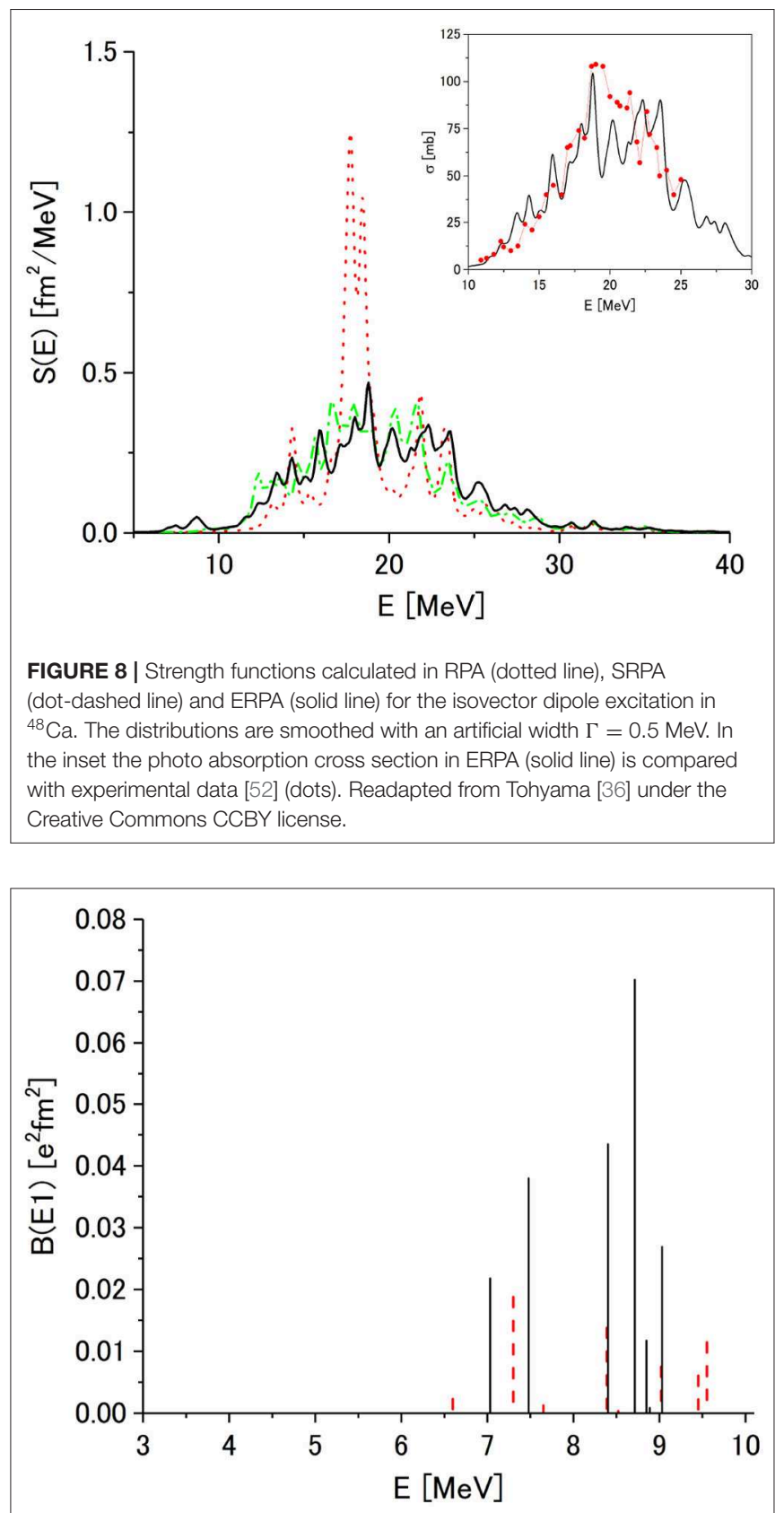

FIGURE 9 | Distribution of $B(E 1)$ strength below $10 \mathrm{MeV}$ calculated in ERPA for ${ }^{48} \mathrm{Ca}$. Experimental data (dashed line) are taken from Hartmann et al. [54]. Adapted from Tohyama [36] under the Creative Commons CCBY license.

the strength distributions in Figure 10 exceed the EWSR value by about $10 \%$ due to the simple approximations for the residual interaction and the continuum states. The main peak in RPA corresponds to the giant quadrupole resonance (GQR). ERPA brings much larger fragmentation of the quadrupole strength than SRPA especially to the low energy region, indicating the importance of the ground-state correlations effects included in ERPA. The large fragmentation of the quadrupole strength is consistent with experiment $[59,60]$. The $\mathrm{p}-\mathrm{p}$ transitions allowed 


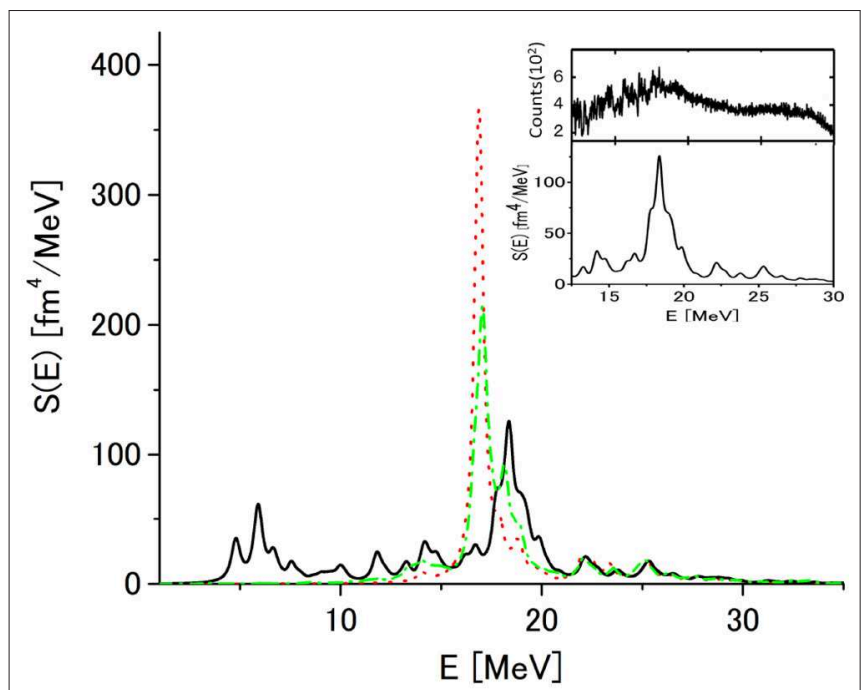

FIGURE 10 | Strength functions calculated in RPA (dotted line), SRPA (dot-dashed line) and ERPA (solid line) for the isoscalor quadrupole excitation in ${ }^{40} \mathrm{Ca}$. The distributions are smoothed with an artificial width $\Gamma=0.5 \mathrm{MeV}$. In the inset the ERPA strength distribution (lower part) is compared with the experimental data from $\left(p, p^{\prime}\right)$ experiments at $E_{p}=200 \mathrm{MeV}$ and $\theta_{\mathrm{Lab}}=11^{\circ}$ [59] (upper part). Redapted from Tohyama [36] under the Creative Commons CCBY license.

by the fractional occupation of the $1 f_{7 / 2}$ states and the coupling of the $2 \mathrm{p}-2 \mathrm{~h}$ amplitudes to the $\mathrm{h}-\mathrm{h}$ or $\mathrm{p}-\mathrm{p}$ amplitudes through $C_{2}$ were found to play an important role in the large fragmentation of the quadrupole strength $[34,36]$. The importance of the coupling of the one-body amplitude to $C_{2}$ in the fragmentation of GQR in ${ }^{40} \mathrm{Ca}$ was also pointed out by the $1 \mathrm{p}-1 \mathrm{~h} \otimes$ phonon configurations model [61, 62]. A large scale SRPA calculation [63] shows a downward shift of the quadrupole strength and larger fragmentation of GQR than the SRPA result in Figure 10. This difference again originates from the difference in the number of the $2 \mathrm{p}-2 \mathrm{~h}$ configurations used. In the inset the ERPA strength distribution in the GQR region (lower part) is compared with the experimental data from $\left(p, p^{\prime}\right)$ experiments [59] (upper part). Although the peak position in ERPA corresponds to the experimental data, ERPA cannot describe the large fragmentation of GQR. The result of the large scale SRPA calculation [63] suggests the importance of higher configurations.

There are 19 sates below $10 \mathrm{MeV}$ in ERPA, which are compared with experiment [54] in Figure 11. The first $2^{+}$state in ${ }^{40} \mathrm{Ca}$ cannot be described in RPA and ERPA because it mainly consists of $4 \mathrm{p}-4 \mathrm{~h}$ states [64] as in the case of ${ }^{16} \mathrm{O}$ [65]. The summed strength below $10 \mathrm{MeV}$ is $166 e^{2} \mathrm{fm}^{4}$ in ERPA, which is about two thirds of the experimental value $263 \pm 46 e^{2} \mathrm{fm}^{4}$ where the first $2^{+}$state is excluded.

From the applications of ERPA to the dipole and quadrupole excitations of ${ }^{48} \mathrm{Ca}$ and ${ }^{40} \mathrm{Ca}$ it was clarified that the ground state correlation effects should be included to explain the large fragmentation of the dipole and quadrupole strengths in doubly-magic nuclei. The ground-state correlation effects in magic nuclei have extensively been studied for spin-isospin modes [31, 50, 66-68].

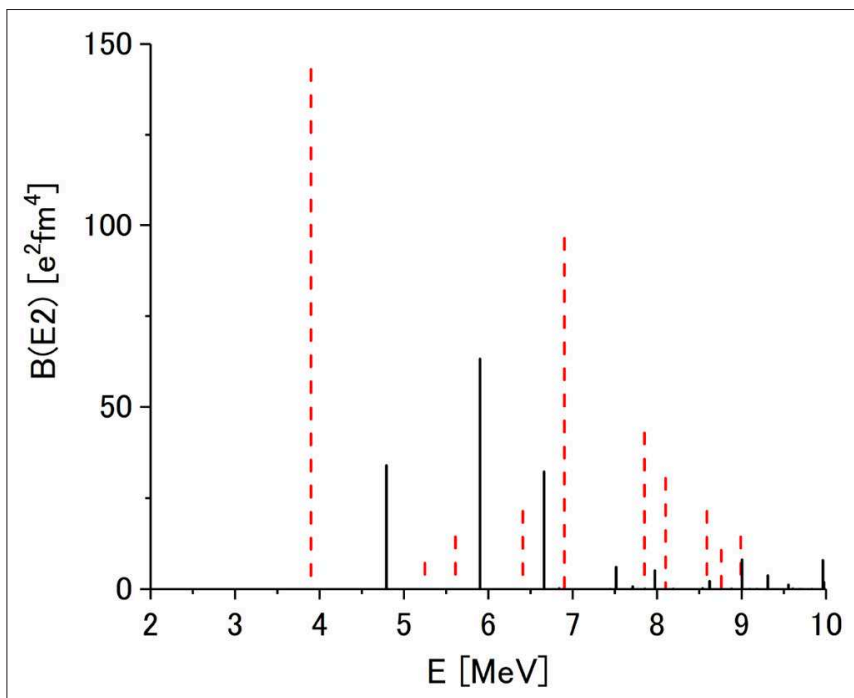

FIGURE 11 | Distribution of $B(E 2)$ strength below $10 \mathrm{MeV}$ for ${ }^{40} \mathrm{Ca}$. Experimental data (dashed line) are taken from Hartmann et al. [54]. Adapted from Tohyama [36] under the Creative Commons CCBY license.

\section{FORMULATION IN TIME-DEPENDENT SINGLE-PARTICLE BASIS}

The first applications of the time-dependent density-matrix approach were based on the TDDM truncation scheme and the TDDM calculations $[6,7]$ were performed by using the time-dependent s.p. wavefunctions obtained from the then available TDHF code with axial symmetry and without spin-orbit force [69]. More advanced TDHF codes with spin-orbit force, unconstrained symmetry and improved effective interactions have been used to solve the TDDM equations $[8,13]$. Since the calculation of the two-body matrix elements is time-consuming, a simpler approximation called $\operatorname{TDDM}^{\mathrm{P}}[13,14]$ has also been employed in heavy-ion collisions, where two-body correlations are considered only for a pair of time reversed s.p. states to reduce the number of matrix elements of $C_{2}$. The TDDM approaches based on the time-dependent s.p. basis have been applied to study the particle transfers in heavy-ion collisions [7], the fusion reactions $[8,9]$ and the damping of giant resonances at zero $[6,70,71]$ and finite temperatures $[10]$. TDDM $^{\mathrm{P}}$ has been applied to the particle transfers in heavy-ion collisions [13] and the fusion reactions [14]. In the following the TDDM formulation in the time-dependent s.p. basis is given and the application to the fusion reactions of ${ }^{16} \mathrm{O}+{ }^{16} \mathrm{O}$ is presented in some detail as an example.

\subsection{TDDM Equations}

The one-body density matrix $\rho$ and the correlated part $C_{2}$ of the two-body density matrix $\rho_{2}$ are expanded with a finite number of time-dependent s.p. states $\psi_{\alpha}$

$$
\rho\left(11^{\prime}, t\right)=\sum_{\alpha \alpha^{\prime}} n_{\alpha \alpha^{\prime}}(t) \psi_{\alpha}(1, t) \psi_{\alpha^{\prime}}^{*}\left(1^{\prime}, t\right),
$$




$$
\begin{aligned}
& C_{2}\left(121^{\prime} 2^{\prime}, t\right)=\rho_{2}-\mathcal{A}(\rho \rho) \\
& =\sum_{\alpha \beta \alpha^{\prime} \beta^{\prime}} C_{\alpha \beta \alpha^{\prime} \beta^{\prime}}(t) \psi_{\alpha}(1, t) \psi_{\beta}(2, t) \psi_{\alpha^{\prime}}^{*}\left(1^{\prime}, t\right) \psi_{\beta^{\prime}}^{*}\left(2^{\prime}, t\right),
\end{aligned}
$$

where the numbers denote space, spin and isospin coordinates. The equations of motion of TDDM in the time-dependent basis consist of the following three coupled equations [6]:

$$
\begin{gathered}
i \frac{\partial}{\partial t} \psi_{\alpha}(1, t)=h(1, t) \psi_{\alpha}(1, t), \\
i \dot{n}_{\alpha \alpha^{\prime}}=\sum_{\beta \gamma \delta}\left[\langle\alpha \beta|v| \gamma \delta\rangle C_{\gamma \delta \alpha^{\prime} \beta}-C_{\alpha \beta \gamma \delta}\left\langle\gamma \delta|v| \alpha^{\prime} \beta\right\rangle\right], \\
i \dot{C}_{\alpha \beta \alpha^{\prime} \beta^{\prime}}=B_{\alpha \beta \alpha^{\prime} \beta^{\prime}}+P_{\alpha \beta \alpha^{\prime} \beta^{\prime}}+H_{\alpha \beta \alpha^{\prime} \beta^{\prime}},
\end{gathered}
$$

where $h$ is the mean-field Hamiltonian given by $\rho$. When the time-dependent s.p. states are chosen, the terms with the s.p. energies on the right-hand side of Equations (5) and (6) are incorporated into the equation for the s.p. wavefunctions [6]. In $\operatorname{TDDM}^{\mathrm{P}}[13,14] H_{\alpha \beta \alpha^{\prime} \beta^{\prime}}$ is neglected and two-body correlations are considered only for a pair of time reversed s.p. states.

\subsection{Fusion Reactions of ${ }^{16} \mathrm{O}+{ }^{16} \mathrm{O}$}

The fusion reactions of ${ }^{16} \mathrm{O}+{ }^{16} \mathrm{O}$ studied in TDDM with the time-dependent s.p. basis are explained below. This work Tohyama and Umar [8] finally solved the longstanding problem of fusion window anomaly. Early TDHF calculations showed that the colliding heavy ions do not fuse in a small impact parameter region when incident energy is higher than a certain relatively low threshold value $E_{\mathrm{th}}$ [2]. This is known as the fusion window anomaly. Experimental search for the fusion window anomaly has found no evidence [72-75]. It was found that the inclusion of spin-orbit force introduced enough one-body dissipation to ${ }^{16} \mathrm{O}+{ }^{16} \mathrm{O}$ collisions [76] because the degeneracy of the $1 p_{3 / 2}$ and $1 p_{1 / 2}$ states is lifted. It was also found [77] that the effects of two-body dissipation taken in TDDM resulted in the doubling of $E_{\text {th }}$ without incorporating spin-orbit force. This is due to the inclusion of additional unoccupied s.p. states in TDDM. In Tohyama and Umar [8] the combined effects of spin-orbit force and two-body dissipation were studied for ${ }^{16} \mathrm{O}+{ }^{16} \mathrm{O}$. In this study the Skyrme II force (SKII) [78] was chosen as an effective interaction to calculate the s.p. wavefunctions since SKII has often been used in TDHF calculations [69]. The s.p. states were restricted to the $1 s-1 p$ and $2 s-1 d$ states and the simple force of the $\delta$ function form $v=v_{0} \delta^{3}\left(\vec{r}-\overrightarrow{r^{\prime}}\right)$ with $v_{0}=-350 \mathrm{MeV} \cdot \mathrm{fm}^{3}$ was used as the residual interaction to facilitate the calculation of the matrix elements. The threshold energy $E_{\text {th }}$ was searched for four different calculation schemes for the head-on collisions of ${ }^{16} \mathrm{O}+{ }^{16} \mathrm{O}$ : TDHF with and without spin-orbit force, and TDDM with and without spin-orbit force.

The obtained results for $E_{\text {th }}$ in the center-of-mass (c.m.) frame are summarized in Table 3. The inclusion of either spinorbit force or two-body dissipation dramatically increases $E_{\text {th }}$.
TABLE 3 | Threshold energy $E_{\text {th }}$ in the center-of-mass frame for the head-on collisions of ${ }^{16} \mathrm{O}+{ }^{16} \mathrm{O}$.

\begin{tabular}{lc}
\hline Method & $\boldsymbol{E}_{\text {th }}[\mathbf{M e V}]$ \\
\hline $\operatorname{TDHF}$ without $\vec{\ell} \cdot \vec{s}$ & 30 \\
$\operatorname{TDDM}$ without $\vec{\ell} \cdot \vec{s}$ & 66 \\
$\operatorname{TDHF}$ with $\vec{\ell} \cdot \vec{s}$ & 69 \\
$\operatorname{TDDM}$ with $\vec{\ell} \cdot \vec{s}$ & 80
\end{tabular}

Fusion occurs below $E_{\text {th. }}$.

However, two-body dissipation increases $E_{\text {th }}$ only about $10 \mathrm{MeV}$ when spin-orbit force is included. It was also found that the translational motion damps faster in TDDM than in TDHF [8] below $E_{\mathrm{c} . \mathrm{m} .}=69 \mathrm{MeV}$ where the colliding system fuses both in TDHF and TDDM. The fusion reactions of ${ }^{16} \mathrm{O}+{ }^{16} \mathrm{O}$ below $E_{\text {th }}$ were also studied by Wen et al. [14] using the $\mathrm{TDDM}^{\mathrm{P}}$ approach and a paring interaction as the residual interaction and it was found that extracted friction coefficients are enhanced by about $20 \%$ due to two-body dissipation.

In the case of heavy-ion collisions the TDDM (and TDDM ${ }^{\mathrm{P}}$ ) equations ostensibly do not conserve the total energy because of the truncation of the s.p. space [7, 14]. Wen et al. [14] has proposed a method to recover the energy conservation within the truncated s.p. space.

\section{SUMMARY AND OUTLOOK}

An approach which extends the time-dependent Hartree-Fock theory (TDHF) based on the equations of motion for reduced density matrices was presented. The equations of motions for reduced density matrices form a coupled chain known as the Bogoliubov-Born-Green-Kirkwood-Yvon (BBGKY) hierarchy. In this time-dependent density-matrix approach (TDDMA) the truncation of the BBGKY hierarchy is applied at the twobody level by approximating the correlated part of the threebody density matrix $\left(C_{3}\right)$. TDDMA has great advantages that a correlated ground state is obtained as a stationary solution of the TDDMA equations and that the small amplitude limit of the TDDMA equations gives the most general form of beyond the random-phase approximation (RPA). TDDMA was applied to the Lipkin model to test the approximations for $C_{3}$. It was found that the simplest approximation where $C_{3}$ is neglected becomes exact in the limits of large number of particles and strong interaction. The extended RPA (ERPA) derived from the TDDMA equations was applied to the one-dimensional Hubbard model to compare with other beyond RPA theories. It was pointed out that when the effects of ground-state correlations are included, the coupling to the two-body amplitudes should also be considered, and vice versa. As the realistic applications of ERPA, the dipole and quadrupole excitations of ${ }^{40} \mathrm{Ca}$ and ${ }^{48} \mathrm{Ca}$ were studied. It was found that the effects of ground-state correlations play an important role in fragmenting the dipole and quadrupole strengths. The TDDMA study for the fusion reactions of ${ }^{16} \mathrm{O}+{ }^{16} \mathrm{O}$ was also presented as an application of the TDDMA formulation with a time-dependent singe-particle 
basis. It was pointed out that the two-body dissipation plays a role in further damping the translational motion of ${ }^{16} \mathrm{O}+{ }^{16} \mathrm{O}$.

Although the obtained results indicate that TDDMA provides a promising beyond mean-field framework to include two-body correlation effects which are missing in TDHF, TDDMA has limitations and issues to be resolved. One limitation is the fact that the number of matrix elements of the two-body density matrix rapidly increases with increasing number of the s.p. states, which forces us to use small s.p. space around the Fermi level in realistic applications, though the obtained results show that basic effects of two-body correlations can be described with rather small s.p. space. In the realistic applications, simple residual interactions of the $\delta$ function form were used to facilitate the calculations of two-body matrix elements whereas the s.p. wavefunctions were obtained from the Skyrme interactions included in TDHF codes. The consistent treatment of the effective interactions is a subject to be addressed in TDDMA.

\section{REFERENCES}

1. Ring P, Schuck P. The Nuclear Many-Body Problem. Berlin: Springer-Verlag (2000).

2. Davies KTR, Devi KRS, Koonin SE, Strayer MR. TDHF calculations of heavyion collisions. In: Bromley DA, editor. Treatise on Heavy Ion Science, Vol. 3. New York, NY: Plenum Press (1985).

3. Sekizawa K. TDHF Theory and its extensions for the multinucleon transfer reaction: a mini review. Front Phys. (2019) 7:20. doi: 10.3389/fphy.2019.00020

4. Bonitz M. Quantum Kinetic Theory, 2nd Edn. Berlin: Springer (2016). doi: 10.1007/978-3-319-24121-0

5. Wang SJ, Cassing W. Explicit treatment of N-body correlations within a density-matrix formalism. Ann Phys. (1985) 159:328-50. doi: 10.1016/0003-4916(85)90116-2

6. Gong M, Tohyama M. Application of a time-dependent densitymatrix formalism. $Z$ Phys A. (1990) 335:153-61. doi: 10.1007/BF012 94470

7. Gong M, Tohyama M, Randrup J. Time-dependent density matrix theory II. Mass dispersion in damped nuclear reactions. Z Phys A. (1990) 335:331-40. doi: 10.1007/BF01304712

8. Tohyama M, Umar AS. Fusion window problem in time-dependent Hartree-Fock theory revisited. Phys Rev C. (2002) 65:037601. doi: 10.1103/PhysRevC.65.037601

9. Tohyama M, Umar AS. Two-body dissipation effects on the synthesis of superheavy elements. Phys Rev C. (2016) 93:034607. doi: 10.1103/PhysRevC.93.034607

10. De Blasio FV, Cassing W, Tohyama M, Bortignon PF, Broglia RA. Nonperturbative study of the damping of giant resonances in hot nuclei. Phys Rev Lett. (1992) 68:1663-6. doi: 10.1103/PhysRevLett.68.1663

11. Pfitzner A, Cassing W, Peter A. Vibrations versus collisions and the iterative structure of two-body dynamics. Nucl Phys A. (1994) 577:753-72. doi: 10.1016/0375-9474(94)90943-1

12. Peter A, Cassing W, Hauser JM, Pfitzner A. Microscopic analysis of two-body correlations in light nuclei. Nucl Phys A. (1994) 573:93-129. doi: 10.1016/0375-9474(94)90016-7

13. Assiee M, Lacroix D. Probing neutron correlations through nuclear breakup. Phys Rev Lett. (2009) 102:202501. doi: 10.1103/PhysRevLett.102.2 02501

14. Wen K, Barton MC, Rios A, Stevenson PD. Two-body dissipation in nuclear fusion reactions. Phys Rev C. (2018) 98:014603. doi: 10.1103/PhysRevC.98.014603

15. Takahara S, Tohyama M, Schuck P. Extended RPA with groundstate correlations in a solvable model. Phys Rev C. (2004) 70:057307. doi: 10.1103/PhysRevC.70.057307
Another point is the truncation scheme of the BBGKY hierarchy itself. In TDDMA the BBGKY hierarchy is truncated at the twobody level by making approximations for $C_{3}$. The truncation violates the properties of reduced density matrices which should be fulfilled if they are derived from an $\mathrm{N}$-body total wavefunction. It was pointed out that the TDDM1 truncation scheme where $C_{3}$ is given by the traced products of the $2 \mathrm{p}-2 \mathrm{~h}$ elements of the two-body correlation matrix largely improves the simple scheme where $C_{3}$ is neglected. However, the validity of TDDM1 cannot be simply extended to highly excited cases such as heavy-ion collisions. The study of the truncation schemes in such cases remains a difficult but interesting subject to be investigated.

\section{AUTHOR CONTRIBUTIONS}

The author confirms being the sole contributor of this work and has approved it for publication.

16. Schmitt K-J, Reinhard P-G, Toepffer C. Truncation of time-dependent many-body theories. Z Phys A. (1990) 336:123-31. doi: 10.1007/BF012 90612

17. Gherega T, Krieg R, Reinhard P-G, Toepffer C. Dynamics of correlations in a solvable model. Nucl Phys A. (1993) 560:166-86. doi: 10.1016/0375-9474(93)90090-K

18. Akbari A, Hashemi MJ, Rubio A, Nieminen RM, van Leeuwen R. Challenges in truncating the hierarchy of time-dependent reduced density matrices equations. Phys Rev B. (2012) 85:23512. doi: 10.1103/PhysRevB.85. 235121

19. Tohyama M. Time-dependent density-matrix approach to collective excitations of a quantum dot. J Phys Soc Jpn. (2012) 81:054707. doi: 10.1143/JPSJ.81.054707

20. Coleman AJ, Yukalov VI. Reduced Density Matrices: Coulson's Challenge. Berlin: Springer-Verlag (2000). doi: 10.1007/978-3-642-58304-9

21. Tohyama M, Schuck P. Truncation scheme of time-dependent densitymatrix approach. Eur Phys J A. (2014) 50:77. doi: 10.1140/epja/i2014-1 4077-x

22. Mazziotti DA. Pursuit of $N$-representability for the contracted Schrödinger equation through density-matrix reconstruction. Phys Rev A. (1999) 60:3618-26. doi: 10.1103/PhysRevA.60.3618

23. Schuck P, Tohyama M. Progress in many-body theory with the equation of motion method: time-dependent density matrix meets self-consistent RPA and applications to solvable models. Phys Rev B. (2016) 93:165117. doi: 10.1103/PhysRevB.93.165117

24. Tohyama M. New truncation scheme for a time-dependent density-matrix approach applied to the ground state of ${ }^{1} 6$ O. Phys Rev C. (2015) 91:017301. doi: 10.1103/PhysRevC.91.017301

25. Lipkin HJ, Meshkov N, Glick AJ. Validity of many-body approximation methods for a solvable model: (I). Exact solutions and perturbation theory. Nucl Phys. (1965) 62:188-98. doi: 10.1016/0029-5582(65) 90862-X

26. Tohyama M, Schuck P. Truncation scheme of time-dependent densitymatrix approach II. Eur Phys J A. (2017) 53:186. doi: 10.1140/epja/i2017-1 2377-3

27. Rowe DJ. Equations-of-Motion method and the extended shell model. Rev Mod Phys. (1968) 40:153. doi: 10.1103/RevModPhys.40.153

28. Rowe DJ. Methods for calculating ground-state correlations of vibrational nuclei. Phys Rev. (1968) 175:1283-92. doi: 10.1103/PhysRev.175.1283

29. Schuck P, Ethofer S. Self-consistent (nuclear) phonons. Nucl Phys A. (1973) 212:269-86. doi: 10.1016/0375-9474(73)90563-0

30. Dukelsky J, Schuck P. Towards a variational theory for RPA-like correlations and fluctuations. Nucl Phys A. (1990) 512:466-82. doi: 10.1016/0375-9474(90)90081-V 
31. Drożdż S, Nishizaki S, Speth J, Wambach J. The nuclear response within extended RPA theories. Phys Rep. (1990) 197:1-65. doi: 10.1016/0370-1573(90)90084-F

32. Litvinova E, Schuck P. Toward an accurate strongly coupled many-body theory with in the equation-of-motion framework. arXiv[Preprint].arXiv:1908.05384.

33. Tohyama M. Role of small-norm components in extended randomphase approximation. Prog Theor Exp Phys. (2017) 2017:093D06. doi: $10.1093 /$ ptep/ptx128

34. Tohyama M. Damping of collective states in an extended random-phase approximation with ground-state correlations. Phys Rev C. (2007) 75:044310. doi: 10.1103/PhysRevC.75.044310

35. Tohyama M. Quadrupole excitation of tin isotopes in extended random-phase approximation. Prog Theor Phys. (2012) 127:1121-30. doi: 10.1143/PTP.127.1121

36. Tohyama M. Effects of ground-state correlations on dipole and quadrupole excitations of ${ }^{4} 0 \mathrm{Ca}$ and ${ }^{4} 8 \mathrm{Ca}$. Prog Theor Exp Phys. (2018) 2018:043D02. doi: $10.1093 /$ ptep/pty035

37. Hubbard J. Electron correlations in narrow energy bands. Proc Roy Soc (London). (1963) 276:238-57. doi: 10.1098/rspa.1963.0204

38. Shavitt I, Bartlett RJ. Many-Body Methods in Chemistry and Physics. Cambridge: Cambridge University Press (2009). doi: 10.1017/CBO9780511596834

39. Cassing W, Pfitzner A. Self-consistent truncation of the BBGKY hierarchy on the two-body level. $Z$ Phys A. (1992) 342:161-7. doi: 10.1007/BF01288464

40. Tohyama M, Schuck P. Extended random-phase approximation with three-body ground-state correlations. Eur Phys J A. (2008) 36:349-57. doi: 10.1140/epja/i2008-10596-3

41. Gell-Mann M, Low F. Bound states in quantum field theory. Phys Rev. (1951) 84:350-4. doi: 10.1103/PhysRev.84.350

42. Tohyama M. Stationary solution of a time dependent density matrix formalism. Prog Theor Phys. (1994) 92:905-8. doi: 10.1143/ptp/92.4.905

43. Tohyama M. Mean-field approach to excitations of a trapped Bose-Einstein condensate gas at finite temperatures. Phys Rev A. (2005) 71:043613. doi: 10.1103/PhysRevA.71.043613

44. Janssen D, Schuck P. On some aspects of selfconsistent RPA theory. Z Phys A. (1991) 339:43-50. doi: 10.1007/BF01282932

45. Tohyama M Schuck P. Truncation scheme of time-dependent density-matrix approach III. Eur Phys J A. (2019) 55:74. doi: 10.1140/epja/i2019-12746-x

46. Jemaï M, Schuck P, Dukelsky J, Bennaceur R. Self-consistent random phase approximation: application to the Hubbard model for finite number of sites. Phys Rev B. (2005) 71:085115. doi: 10.1103/PhysRevB.71.085115

47. Beiner M, Flocard H, Van Giai N, Quentin P. Nuclear groundstate properties and self-consistent calculations with the skyrme interaction: (I). Spherical description. Nucl Phys A. (1975) 238:29-69. doi: 10.1016/0375-9474(75)90338-3

48. Lee J, Tostevin JA, Brown BA, Delaunay F, Lynch WG, Saelim MJ, et al. Reduced neutron spectroscopic factors when using potential geometries constrained by Hartree-Fock calculations. Phys Rev C. (2006) 73:044608. doi: 10.1103/PhysRevC.73.044608

49. Agassi D, Gillet V, Lumbroso A. RPA description of the ground state of ${ }^{1} 2 \mathrm{C},{ }^{1} 6 \mathrm{O},{ }^{4} 0 \mathrm{Ca}$ and ${ }^{2} 08 \mathrm{~Pb}$. Nucl Phys A. (1969) 130:129-45. doi: 10.1016/0375-9474(69)90966-X

50. Adachi S, Lipparini E, Nguyen van Giai. Gamow-Teller and M1 strengths in closed-shell nuclei. Nucl Phys A. (1985) 438:1-14. doi: 10.1016/0375-9474(85)90115-0

51. Tohyama M, Schuck P. Odd particle number random-phase approximation and extensions: applications to particle and hole states around ${ }^{1} 6 \mathrm{O}$. Phys Rev C. (2013) 87:044316. doi: 10.1103/PhysRevC.87.044316

52. O'Keefe GJ, Thompson MN, Assafiri YI, Pywell RE. The photonuclear cross sections of ${ }^{4} 8 \mathrm{Ca}$. Nucl Phys A. (1987) 469:239-52. doi: 10.1016/0375-9474(87)90108-4

53. Gambacurta D, Grasso M, Catara F. Collective nuclear excitations with Skyrme-second random-phase approximation. Phys Rev C. (2010) 81:054312. doi: 10.1103/PhysRevC.81.054312

54. Hartmann T, Enders J, Mohr P, Vogt K, Volz S, Zilges A. Measurement of the dipole and electric quadrupole strength distributions up to $10 \mathrm{MeV}$ in the doubly magic nuclei ${ }^{4} 0 \mathrm{Ca}$ and ${ }^{4} 8 \mathrm{Ca}$. Phys Rev Lett. (2000) 85:274-7. doi: 10.1103/PhysRevLett.85.274

55. Gambacurta D, Grasso M, Catara F. Low-lying dipole response in the stable ${ }^{4} 0,48 \mathrm{Ca}$ nuclei with the second random-phase approximation. Phys Rev C. (2011) 84:034301. doi: 10.1103/PhysRevC.84.034301

56. Gambacurta D, Grasso M, Vasseur O. Electric dipole strength and dipole polarizability in ${ }^{4} 8 \mathrm{Ca}$ within a fully self-consistent second random-phase approximation. Phys Lett B. (2018) 777:163-8. doi: 10.1016/j.physletb.2017.12.026

57. Hartmann T, Babilon M, Kamerdzhiev S, Litvinova E, Savran D, Volz S, et al. Microscopic nature of the pygmy dipole resonance: the stable Ca isotopes. Phys Rev Lett. (2004) 93:192501. doi: 10.1103/PhysRevLett.93.192501

58. Egorova I A, Litvinova E. Electric dipole response of neutron-rich calcium isotopes in relativistic quasiparticle time blocking approximation. Phys Rev C. (2016) 94:034322. doi: 10.1103/PhysRevC.94.034322

59. Usman I, Buthelezia Z, Carterb J, Cooper GRJ, Fearickd RW, Förtsch $\mathrm{SV}$, et al. Fine structure of the isoscalar giant quadrupole resonance in ${ }^{4} 0 \mathrm{Ca}$ due to Landau damping? Phys Lett B. (2011) 698:191-5. doi: 10.1016/j.physletb.2011.03.015

60. Diesener H, Helm U, Herbert G, Huck V, von Neumann-Cosel P, Rangacharyulu $\mathrm{C}$, et al. Excitation and decay of giant resonances in the ${ }^{4} 0 \mathrm{Ca}\left(e, e^{\prime} x\right)$ reaction. Phys Rev Lett. (1994) 72:1994-7. doi: 10.1103/PhysRevLett.72.1994

61. Kamerdzhiev S, Speth J, Tertychny G. Splitting of the isoscalar E2 giant resonance and evidence of low-lying E0 strength in ${ }^{4} 0 \mathrm{Ca}$. Phys Rev Lett. (1995) 74:3943-6. doi: 10.1103/PhysRevLett.74.3943

62. Kamerdzhiev S, Speth J, Tertychny G. Microscopic description of the E0, E2 and E1 giant resonances in ${ }^{4} 0 \mathrm{Ca},{ }^{4} 8 \mathrm{Ca}$ and ${ }^{5} 6 \mathrm{Ni}$. Nucl Phys A. (1997) 624:328-48. doi: 10.1016/S0375-9474(97)00315-1

63. Vasseur O, Gambacurta D, Grasso M. Systematic study of giant quadrupole resonances with the subtracted second random-phase approximation: Beyond-mean-field centroids and fragmentation. Phys Rev C. (2018) 98:044313. doi: 10.1103/PhysRevC.98.044313

64. Caurier E, Menéndez J, Nowacki F, Poves A. Coexistence of spherical states with deformed and superdeformed bands in doubly magic ${ }^{4} 0 \mathrm{Ca}$ : a shell-model challenge. Phys Rev C. (2007) 75:054317. doi: 10.1103/PhysRevC.75.054317

65. Zuker AP, Buck B, McGrory JB. Structure of $\mathrm{O}^{1} 6$. Phys Rev Lett. (1968) 21:39-43. doi: 10.1103/PhysRevLett.21.39

66. Gross W, Meuer D, Richter A, Spamer E, Titze O, Knüpfer W. A strong magnetic dipole excitation in ${ }^{4} 0 \mathrm{Ca}$ observed in highresolution inelastic electron scattering and coherent spin-flip transitions due to cground-state correlations. Phys Lett B. (1979) 84:296-300. doi: 10.1016/0370-2693(79)90043-1

67. Takayanagi K, Shimizu K, Arima A. A theoretical study of magnetic dipole transitions in ${ }^{4} 8 \mathrm{Ca}$. Nucl Phys A. (1988) 481:313-32. doi: 10.1016/0375-9474(88)90499-X

68. Robin C, Litvinova E. Time-reversed particle-vibration loops and nuclear Gamow-Teller response. Phys Rev Lett. (2019) 123:202501. doi: 10.1103/PhysRevLett.123.202501

69. Davies KTR, Koonin SE. Skyrme-force time-dependent Hartree-Fock calculations with axial symmetry. Phys Rev C. (1981) 23:2042-61. doi: 10.1103/PhysRevC.23.2042

70. Tohyama M, Umar AS. Dipole resonances in oxygen isotopes in time-dependent density-matrix theory. Phys Lett B. (2001) 516:415-9. doi: 10.1016/S0370-2693(01)00925-X

71. Tohyama M, Umar AS. Quadrupole resonances in unstable oxygen isotopes in time-dependent density-matrix formalism. Phys Lett B. (2002) 549:72-8. doi: 10.1016/S0370-2693(02)02885-X

72. Lazzarini A, Doubre H, Lesko KT, Metag V, Seamster A, Vandenbosch R, et al. Search for a fusion $L$ window in the ${ }^{1} 6 \mathrm{O}+{ }^{1} 6 \mathrm{O}$ system at $E_{c . m} .=34 \mathrm{MeV}$. Phys Rev C. (1981) 24:309-12. doi: 10.1103/PhysRevC.24.309

73. Kox S, Cole AJ, Ost R. Determination of limiting angular momenta for fusion from statistical-model fits to mass distributions of evaporation residues. Phys Rev Lett. (1980) 44:1204-6. doi: 10.1103/PhysRevLett.44.1204

74. Szanto de Toledo A, Cormier TM, Herman M, Lin B, Stwertka PM, Coimbra $\mathrm{MM}$, et al. Limiting angular momenta for light heavy-ion fusion at high energy. Phys Rev Lett. (1981) 47:1881-4. doi: 10.1103/PhysRevLett.47.1881 
75. Ikezoe H, Shikazono N, Tomita Y, Ideno K, Sugiyama Y, Takekoshi E, et al. Measurements of evaporation residues to investigate lower limiting angular momenta in fusion reactions. Nucl Phys A. (1986) 456:298-316. doi: 10.1016/0375-9474(86)90395-7

76. Umar AS, Strayer MR, Reinhard P-G. Resolution of the fusion window anomaly in heavy-ion collisions. Phys Rev Lett. (1986) 56:2793-6. doi: 10.1103/PhysRevLett.56.2793

77. Tohyama M. Application of quantum theory of particle collisions to ${ }^{1} 6 \mathrm{O}+$ ${ }^{1} 6$ O reactions. Phys Rev C. (1987) 36:187-92. doi: 10.1103/PhysRevC.36.187

78. Vautherin D, Brink DM. Hartree-Fock calculations with Skyrme's interaction. I. Spherical nuclei. Phys Rev C. (1972) 5:626-47. doi: 10.1103/PhysRevC.5.626
Conflict of Interest: The author declares that the research was conducted in the absence of any commercial or financial relationships that could be construed as a potential conflict of interest.

Copyright (๑ 2020 Tohyama. This is an open-access article distributed under the terms of the Creative Commons Attribution License (CC BY). The use, distribution or reproduction in other forums is permitted, provided the original author(s) and the copyright owner(s) are credited and that the original publication in this journal is cited, in accordance with accepted academic practice. No use, distribution or reproduction is permitted which does not comply with these terms. 